Article

\title{
Thermodynamic Database for Mg Alloys-Progress in Multicomponent Modeling
}

\section{Rainer Schmid-Fetzer * and Joachim Gröbner}

Institute of Metallurgy, Clausthal University of Technology, Robert-Koch-Str. 42, D-38678

Clausthal-Zellerfeld, Germany; E-Mail: joachim.groebner@tu-clausthal.de

* Author to whom correspondence should be addressed; E-Mail: schmid-fetzer@tu-clausthal.de; Tel.: +49-5323-72-2150; Fax: +49-5323-72-3120.

Received: 2 July 2012; in revised form: 20 August 2012 / Accepted: 21 August 2012 /

Published: 14 September 2012

\begin{abstract}
Progress in systematic development of a thermodynamic database for $\mathrm{Mg}$ alloys with 21 components is reported. Models for multicomponent alloys are built in a methodical approach from quantitative descriptions of unary, binary and ternary subsystems. For a large number of ternary - and some higher-alloy systems, an evaluation of the modeling depth is made with concise reference to experimental work validating these thermodynamic descriptions. A special focus is on ternary intermetallic phase compositions. These comprise solutions of the third component in a binary compound as well as truly ternary solid solution phases, in addition to the simple ternary stoichiometric phases. Concise information on the stability ranges is given. That evaluation is extended to selected quaternary and even higher alloy systems. Thermodynamic descriptions of intermetallic solution phases guided by their crystal structure are also elaborated and the diversity of such unified phases is emphasized.
\end{abstract}

Keywords: multicomponent simulation; computational thermodynamics; phase formation

\section{Introduction}

Thermodynamic simulation of phase formation and calculated phase diagrams have been successfully used as an effective tool in focused alloy design and process optimization of magnesium alloys [1-4]. While the calculated phase diagrams provide a comprehensive overview of the equilibrium phase assembly, more quantitative details are obtained for simulations at a fixed alloy 
composition. Even for really multicomponent alloys, far beyond ternary systems, easily interpreted diagrams are obtained for the phase fractions and compositions with varying temperature. Often the as-cast state is reasonably approximated by simulations under Scheil conditions, whereas the other limiting case, complete equilibrium, provides the phases expected after long heat treatment. Thus, the temperature window for solution heat treatment for that specific multicomponent alloy can be predicted and the expected phase transformations, starting from the as-cast state, are revealed. It is evident that the quality of the underlying thermodynamic database is decisive for the success of such applications.

Such thermodynamic databases for multicomponent and multiphase alloys are developed using the Calphad approach [5,6], and comprise all possible phases in the alloy system, including the liquid phase and other complex solution phases. The present work will focus on specific aspects of a thermodynamic $\mathrm{Mg}$ alloy database, which is developed in an ongoing effort in the authors' group since the mid 1990's [7] by experiments combined with Calphad modeling as sketched in Figure 1. Some general aspects concerning modeling formalism, quality assurance and applications to multicomponent systems were published in 2001 [8], 2005 [9] and 2008 [10].

Figure 1. Milestones during the Mg alloy database development.

\begin{tabular}{|c|c|}
\hline Today & $\begin{array}{l}\text { Progress on Mg-RE \& other advanced alloys ongoing } \\
\text { DB: } 21 \text { components, } 445 \text { phases, } \\
\text { Extending \& improving multicomponent interactions \& solid solutions }\end{array}$ \\
\hline \multirow[t]{2}{*}{2008} & $\begin{array}{l}\text { Progress on advanced alloys published } J \text { Metals } \\
\text { DB: } 20 \text { components, } 412 \text { phases, } 134 \text { binaries, } 75 \text { ternaries, } 11 \text { quat. }\end{array}$ \\
\hline & 2 \\
\hline \multirow[t]{2}{*}{2005} & $\begin{array}{l}\text { Progress } \& \text { Quality assurance published Adv. Eng. Mater. } \\
\text { DB: } 17 \text { components, } 285 \text { phases, } 97 \text { binaries, } 37 \text { ternaries, } 7 \text { quat. }\end{array}$ \\
\hline & 2 \\
\hline \multirow[t]{2}{*}{2001} & $\begin{array}{l}\text { First summary } \& \text { Applications published Adv. Eng. Mater. } \\
\text { DB: } 13 \text { components, } 139 \text { phases, } 59 \text { binaries, } 17 \text { ternaries }\end{array}$ \\
\hline & 2 \\
\hline 1995 & Focused work on Mg alloys started (Experiments \& Modeling) \\
\hline
\end{tabular}

The major components of the database are given in Figure 2 in a clockwise arrangement from the more conventional to the more advanced alloy systems. The most common alloying systems comprise aluminum (A), zinc (Z) and some manganese (M) in the AZ group or, without zinc, in the AM alloy group. The AS and AL series contain Si (S) or Li (L), in addition to Al. More advanced developments include $\mathrm{Ca}(\mathrm{X}), \mathrm{Sr}(\mathrm{J}), \mathrm{Sn}(\mathrm{T}), \mathrm{Y}(\mathrm{W})$ or the important rare earths elements (E) in combinations such as the AXJ, ZE or WZ alloys.

With growing size, the key issues arising are consistency, coherency and quality assurance. These issues also concern extension, maintenance and updating of the database. These specific issues and the application by predicting phase formation during solidification and heat treatment in multicomponent magnesium alloys from thermodynamic calculations are addressed in a recent work [11]. The purpose of the present work is to reveal the necessity of meticulously describing the thermodynamics of intermetallic solid solution phases at least up to the ternary alloy systems. It will be shown that these are abundant in $\mathrm{Mg}$ alloy systems and that simplified descriptions focusing on stoichiometric binary or ternary phases must fail dramatically. That aspect is also worked out for the proper thermodynamic modeling of intermetallic solution phases containing many more than three components. In addition to 
the database, a thermodynamic software package based on the principle of minimizing the Gibbs energy of the multiphase system is required to perform the actual calculations, such as Thermo-calc [12], FactSage [13] or Pandat [14]. In the present work, the software Pandat was used for all calculations.

Figure 2. Most important chemical components of the database. Additionally, smaller single characters indicate the standard system of alloy designation according to ASTM B 275 for Mg alloys.

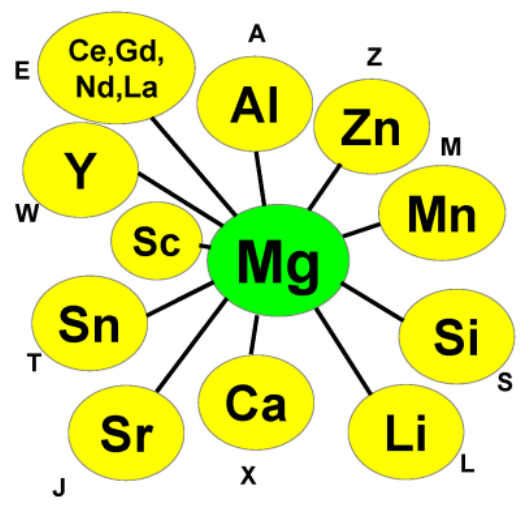

\section{Investigated Binary Alloy Systems}

The database is built up systematically, from unary to binary to ternary and higher systems. The thermodynamic descriptions for the elements are taken from the unary SGTE data compiled by Dinsdale [15].

The 20 main components in the database are $\mathrm{Ag}, \mathrm{Al}, \mathrm{Ca}, \mathrm{Ce}, \mathrm{Cu}, \mathrm{Fe}, \mathrm{Gd}, \mathrm{La}, \mathrm{Li}, \mathrm{Mg}, \mathrm{Mn}, \mathrm{Nd}, \mathrm{Ni}$, $\mathrm{Sc}, \mathrm{Si}, \mathrm{Sn}, \mathrm{Sr}, \mathrm{Y}, \mathrm{Zn}$ and Zr. They form a total of 190 possible binary systems. An overview of our critical assessment concerning the Calphad-type modeling status of these binary systems is given in Table 1. All color-marked systems (green/yellow) are modeled in the complete composition range. It should be emphasized that in the process of Calphad modeling, all the experimental data of that binary system are put on the table: phase equilibria and phase boundaries, as well as thermodynamic data, such as enthalpies, specific heat, partial pressures or chemical activities. A self-consistent description of all these data, including the calculated phase diagram, is then produced through models of the Gibbs energy for each phase in the system. Thus, the binary systems marked green in Table 1 (status A, reliable description) are also very well validated by the entity of experimental data.

Less reliable descriptions (status B) are marked yellow. That indicates either scant experimental data or larger deviations between the thermodynamic calculations and the experimental data. Blank fields indicate systems that are not modeled yet (status C). That means that the Gibbs energies of the terminal solution phases, emerging from the elements, are extrapolated, assuming ideal solutions. For example, the calculated Ag-Sr phase diagram shows only the solution phases Liquid, FCC and BCC in this extrapolation but no intermetallic phase. Similarly, the Ag-Fe phase diagram will show complete solubility of the solution phases Liquid, FCC and BCC, but not the miscibility gap. 
Table 1. Classification of the modeling status of basic binary systems.

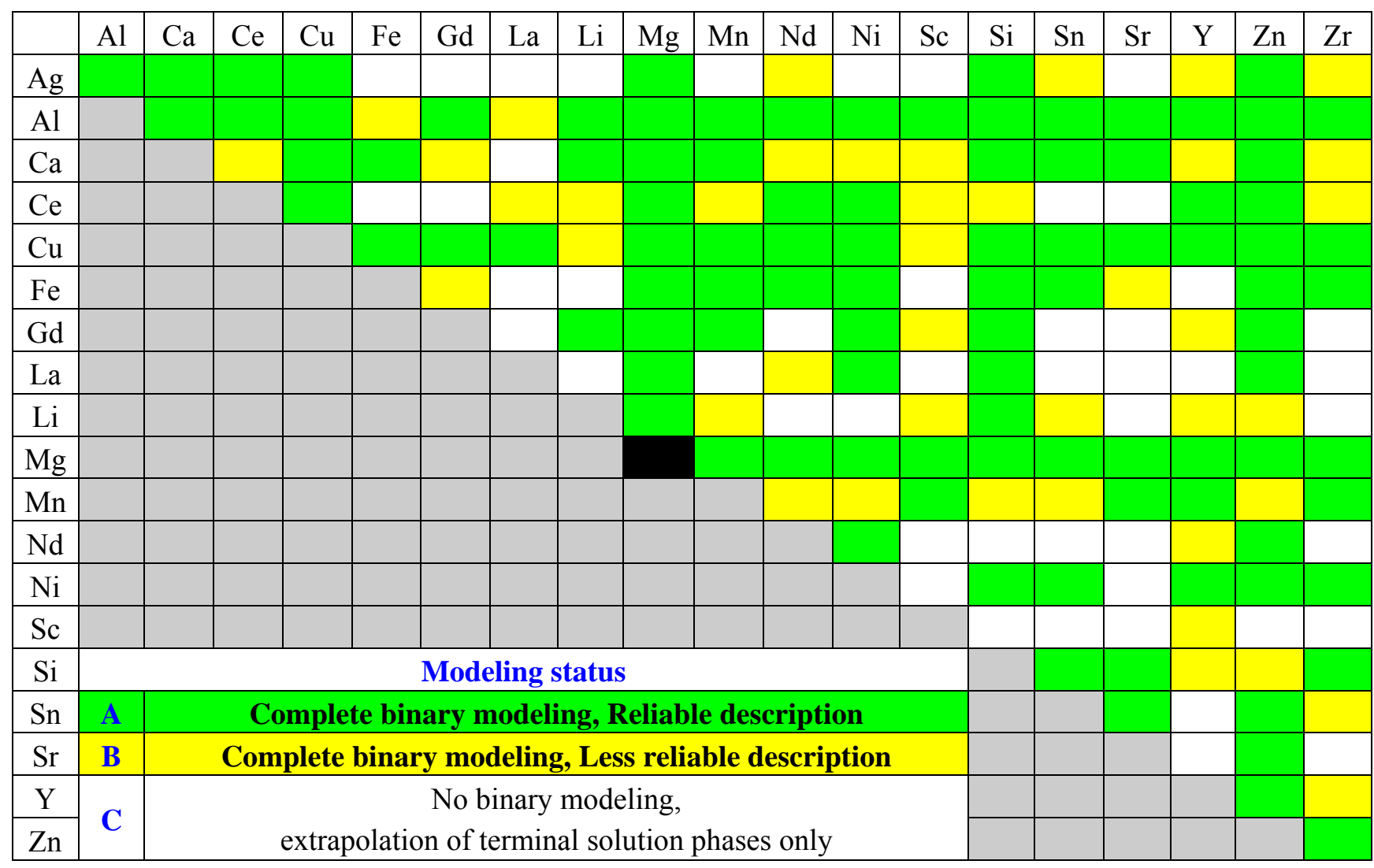

Carbon is not included in Table 1 but in the database, where the two additional binary carbon systems (Al-C, C-Ca) are modeled with status B and two (C-Mg, C-Si) with status A.

For any application to multicomponent $\mathrm{Mg}$ alloys, it is important to also consider the binary systems containing no $\mathrm{Mg}$. For example, in ternary $\mathrm{Mg}-\mathrm{C}-\mathrm{Si}$ alloys, the description of the binary system $\mathrm{C}$-Si is of utmost importance because of the very stable compound $\mathrm{SiC}$. This phase precipitates dominantly over a very wide range of conditions from $\mathrm{Mg}-\mathrm{C}-\mathrm{Si}$ alloys, in addition to $\mathrm{Mg}_{2} \mathrm{Si}$ or graphite. This is just one example why the secondary phases found in multicomponent $\mathrm{Mg}$ alloys should never be read from binary $\mathrm{Mg}$-X phase diagrams alone. More detailed comments to the 146 binary systems modeled in the database (status A or B) are beyond the scope of this summary.

\section{Investigated Ternary and Multicomponent Alloy Systems}

A widely used approach in calculating phase diagrams of ternary and multicomponent systems is to start with the Calphad-descriptions of the binary subsystems and to extrapolate these into the higher system. That often works reasonably well as a first approximation if the proper extrapolation scheme for the binary terminal solution phases is used, such as Liquid, $\mathrm{HCP}(\mathrm{Mg})$ and others extending from a pure component [6]. It is also supposed that ternary or higher compounds are less abundant than binary compounds. Ternary interaction parameters are then considered as a refinement only. That gives rise to the rule of thumb: the more components that are included, the less additional information is required. Even though this is generally adequate, it will be shown below that certainly for $\mathrm{Mg}$ alloys due diligence is required. The main reason is that intermetallic solid solution phases extending to ternary or 
higher alloy systems are abundant in Mg alloys. Therefore, calculations that are not based on proper thermodynamic descriptions of at least the important ternary systems may be precarious.

The ternary systems implemented in the database with Calphad-type modeling of the complete composition range are summarized in Table 2 for the 53 modeled ternary $\mathrm{Mg}-\mathrm{X}-\mathrm{Z}$ systems and for the additional 46 non-Mg systems in Table 3. Concerning the experimental validation, the same assessment as for the binary systems applies. The ternary systems marked with green color in Tables 2 and 3 (status A) are well validated by the entity of experimental data, whereas the less reliable descriptions (status B) are marked yellow. The "year of study" is important information, since the experimental literature for each system up to the year of publication is summarized and critically assessed in the reference given. The key aspect worked out in the present work concerns the solid phases occurring at compositions within each ternary system as stoichiometric or solution phases.

Table 2. Ternary $\mathrm{Mg}$ systems with complete thermodynamic descriptions and their classified modeling status. In ternary intermetallic solid solution phases extending from a binary phase with limited solubility of the third component, the majority component is marked in bold font.

\begin{tabular}{|c|c|c|c|c|c|}
\hline System & $\begin{array}{l}\text { Modeling } \\
\text { status }\end{array}$ & $\begin{array}{l}\text { Ternary intermetallic } \\
\text { solid solution phases }\end{array}$ & $\begin{array}{l}\text { Ternary } \\
\text { stoichiometric phases }\end{array}$ & $\begin{array}{l}\text { Year of } \\
\text { study }\end{array}$ & Ref. \\
\hline $\mathrm{Mg}-\mathrm{Ag}-\mathrm{Al}$ & B & $\operatorname{Ag}(\mathrm{Al}, \mathbf{M g})_{3}, \operatorname{Ag}(\mathrm{Al}, \mathbf{M g})_{4}$ & $\mathrm{Al}_{4} \mathrm{GdMg}$ & 1997 & {$[16]$} \\
\hline $\mathrm{Mg}-\mathrm{Ag}-\mathrm{Cu}$ & B & none & - & 1997 & {$[16]$} \\
\hline $\mathrm{Mg}-\mathrm{Al}-\mathrm{Ca}$ & A & $\begin{array}{l}\mathrm{C} 14-\mathrm{Ca}(\mathbf{M g}, \mathrm{Al})_{2} \\
\mathrm{C} 15-\mathrm{Ca}(\mathrm{Al}, \mathrm{Mg})_{2} \\
* \mathrm{C} 36-\mathrm{Ca}(\mathrm{Al}, \mathrm{Mg})_{2} \\
\mathrm{Ca}_{8}(\mathbf{A l}, \mathrm{Mg})_{3}\end{array}$ & - & 2009 & {$[17,18]$} \\
\hline $\mathrm{Mg}-\mathrm{Al}-\mathrm{Ce}$ & A & $\begin{array}{l}\mathrm{Ce}(\mathbf{M g}, \mathrm{Al}) ; \mathrm{Ce}(\mathbf{M g}, \mathrm{Al})_{12} ; \\
\mathrm{Ce}(\mathrm{Mg}, \mathrm{Al})_{2} \\
{ }^{*} \mathrm{C} 14-\mathrm{Cu}(\mathrm{Mg}, \mathrm{Al})_{2}\end{array}$ & $\begin{array}{l}\mathrm{Al}_{13} \mathrm{CeMg}_{6} \\
\mathrm{Al}_{7} \mathrm{Cu}_{3} \mathrm{Mg}_{6}\end{array}$ & 2002 & {$[19]$} \\
\hline $\mathrm{Mg}-\mathrm{Al}-\mathrm{Cu}$ & $\mathrm{A}$ & $\begin{array}{l}\mathrm{C} 15-\mathrm{Cu}(\mathrm{Al}, \mathrm{Mg})_{2} \\
{ }^{*} \mathrm{C} 36-\mathrm{Cu}(\mathrm{Al}, \mathrm{Mg})_{2}\end{array}$ & $\begin{array}{l}\mathrm{Al}_{3} \mathrm{CuMg} \\
\mathrm{V}-\mathrm{Al}_{5} \mathrm{Cu}_{6} \mathrm{Mg}_{2}\end{array}$ & 1998 & {$[20]$} \\
\hline Mg-Al-Gd & A & - & $\mathrm{Al}_{4} \mathrm{GdMg}$ & 2001 & {$[21]$} \\
\hline Mg-Al-Li & A & $\gamma \mathrm{Mg}_{17}(\mathbf{A l}, \mathrm{Li})_{12} ; \mathrm{Li}(\mathbf{A l}, \mathrm{Mg})$ & $\mathrm{Al}_{53} \mathrm{Li}_{33} \mathrm{Mg}_{14}$ & 2001 & {$[22]$} \\
\hline Mg-Al-Mn & A & - & $\mathrm{Al}_{18} \mathrm{Mg}_{3} \mathrm{Mn}_{2}$ & 2007 & {$[23,24]$} \\
\hline $\mathrm{Mg}-\mathrm{Al}-\mathrm{Sc}$ & A & $\begin{array}{l}\mathrm{Sc}(\mathbf{A l}, \mathrm{Mg}) ; \operatorname{Sc}(\mathbf{A l}, \mathrm{Mg})_{2} \\
\operatorname{Sc}(\mathbf{A l}, \mathrm{Mg})_{3}\end{array}$ & - & 1999 & {$[25]$} \\
\hline $\mathrm{Mg}-\mathrm{Al}-\mathrm{Si}$ & $\mathrm{A}$ & $\begin{array}{l}{ }^{*} \mathrm{C} 36-(\mathrm{Al}, \mathrm{Mg}, \mathrm{Si})(\mathrm{Al}, \mathrm{Mg}, \mathrm{Si})_{2} \\
\operatorname{Sr}(\mathbf{M g}, \mathrm{Al})_{2} ; \mathrm{Sr}_{6}(\mathbf{M g}, \mathrm{Al})_{23}\end{array}$ & - & 2001 & {$[22]$} \\
\hline $\mathrm{Mg}-\mathrm{Al}-\mathrm{Sr}$ & $\mathrm{A}$ & $\begin{array}{l}\mathrm{Sr}_{9}(\mathbf{M g}, \mathrm{Al})_{38} ; \mathrm{Sr}_{2}(\mathbf{M g}, \mathrm{Al})_{17} ; \\
\operatorname{Sr}(\mathbf{A l}, \mathrm{Mg})_{2} ; \operatorname{Sr}(\mathbf{A l}, \mathrm{Mg})_{4}\end{array}$ & $\mathrm{Al}_{38} \mathrm{Mg}_{58} \mathrm{Sr}_{4}$ & 2007 & {$[26]$} \\
\hline $\mathrm{Mg}-\mathrm{Al}-\mathrm{Sn}$ & $\mathrm{A}$ & $\begin{array}{l}\text { none } \\
\beta \mathrm{Mg}_{2}(\mathbf{A l}, \mathrm{Zn})_{3} ; \gamma \mathrm{Mg}_{17}(\mathbf{A l}, \mathrm{Zn})_{12}\end{array}$ & - & 2007 & {$[27]$} \\
\hline Mg-Al-Zn & A & $\begin{array}{l}(\mathbf{M g}, \mathrm{Al}) \mathrm{Zn} ;(\mathbf{M g}, \mathrm{Al})_{2} \mathrm{Zn}_{3} ; \\
\mathrm{C} 14-(\mathrm{Mg}, \mathrm{Al}) \mathrm{Zn}_{2} ;(\mathbf{M g}, \mathrm{Al})_{2} \mathrm{Zn}_{11} ; \\
{ }^{*} \theta-\mathrm{AlMgZn} ;{ }^{*} \tau-\mathrm{AlMgZn}\end{array}$ & - & 2006 & {$[28]$} \\
\hline
\end{tabular}


Table 2. Cont.

\begin{tabular}{|c|c|c|c|c|c|}
\hline System & $\begin{array}{l}\text { Modeling } \\
\text { status }\end{array}$ & $\begin{array}{l}\text { Ternary intermetallic } \\
\text { solid solution phases }\end{array}$ & $\begin{array}{l}\text { Ternary } \\
\text { stoichiometric phases }\end{array}$ & $\begin{array}{l}\text { Year of } \\
\text { study }\end{array}$ & Ref. \\
\hline $\mathrm{Mg}-\mathrm{Ca}-\mathrm{Ce}$ & $\mathrm{A}$ & $\mathrm{Ce}_{5}(\mathbf{M g}, \mathrm{Ca})_{41}$ & - & 2007 & [29] \\
\hline $\mathrm{Mg}-\mathrm{Ca}-\mathrm{Li}$ & $\mathrm{A}$ & $\mathrm{C} 14-\mathrm{Ca}(\mathrm{Mg}, \mathrm{Li})_{2}$ & - & 2002 & {$[30]$} \\
\hline $\mathrm{Mg}-\mathrm{Ca}-\mathrm{Si}$ & $\mathrm{A}$ & ${ }^{*} \mathrm{Ca}(\mathrm{Ca}, \mathrm{Mg}) \mathrm{Si}$ & $\mathrm{Ca}_{7} \mathrm{Mg}_{6} \mathrm{Si}_{14}$ & 2003 & {$[31]$} \\
\hline $\mathrm{Mg}-\mathrm{Ca}-\mathrm{Sn}$ & A & $(\mathrm{Ca}, \mathrm{Mg}) \mathrm{CaSn}$ & - & 2011 & {$[32]$} \\
\hline $\mathrm{Mg}-\mathrm{Ca}-\mathrm{Sr}$ & A & $\mathrm{C} 14-(\mathrm{Ca}, \mathrm{Sr}) \mathrm{Mg}_{2}$ & - & 2009 & [33] \\
\hline $\mathrm{Mg}-\mathrm{Ca}-\mathrm{Zn}$ & A & $\begin{array}{l}\mathrm{C} 14-\mathrm{Ca}(\mathbf{M g}, \mathrm{Zn})_{2} \\
(\mathrm{Ce}, \mathrm{La}) \mathrm{Mg}\end{array}$ & $\mathrm{Ca}_{2} \mathrm{Mg}_{6} \mathrm{Zn}_{3}$ & 2004 & {$[34]$} \\
\hline $\mathrm{Mg}-\mathrm{Ce}-\mathrm{La}$ & A & $\begin{array}{l}(\mathrm{Ce}, \mathrm{La}) \mathrm{Mg}_{3} ;(\mathbf{C e}, \mathrm{La})_{5} \mathrm{Mg}_{41} \\
(\mathrm{Ce}, \mathrm{La}) \mathrm{Mg}_{12} ;(\mathrm{Ce}, \mathbf{L a})_{2} \mathrm{Mg}_{17}\end{array}$ & - & 2012 & [35] \\
\hline $\mathrm{Mg}-\mathrm{Ce}-\mathrm{Nd}$ & $\mathrm{A}$ & $\begin{array}{l}(\mathrm{Ce}, \mathrm{Nd}) \mathrm{Mg} ;(\mathrm{Ce}, \mathrm{Nd}) \mathrm{Mg}_{3} \\
(\mathrm{Ce}, \mathrm{Nd})_{5} \mathrm{Mg}_{41} ;(\mathbf{C e}, \mathrm{Nd}) \mathrm{Mg}_{12}\end{array}$ & - & 2011 & {$[36]$} \\
\hline $\mathrm{Mg}-\mathrm{Ce}-\mathrm{Sn}$ & A & & $\mathrm{MgCeSn}$ & 2012 & {$[37]$} \\
\hline $\mathrm{Mg}-\mathrm{Ce}-\mathrm{Y}$ & $\mathrm{B}$ & $\begin{array}{l}(\mathbf{C e}, \mathrm{Y}) \mathrm{Mg} ;(\mathbf{C e}, \mathrm{Y}) \mathrm{Mg}_{2} ; \\
(\mathbf{C e}, \mathrm{Y}) \mathrm{Mg}_{3} ;(\mathbf{C e}, \mathrm{Y})_{5} \mathrm{Mg}_{41} ; \\
(\mathbf{C e}, \mathrm{Y}) \mathrm{Mg}_{12} ;(\mathbf{Y}, \mathrm{Ce}) \mathrm{Mg}_{2} ; \\
(\mathbf{Y}, \mathrm{Ce})_{5} \mathrm{Mg}_{24} ; *(\mathrm{Ce}, \mathrm{Y}) \mathrm{Mg}_{5}\end{array}$ & - & 2010 & {$[38]$} \\
\hline $\mathrm{Mg}-\mathrm{Ce}-\mathrm{Zn}$ & $\mathrm{A}$ & $\begin{array}{l}(\mathbf{C e}, \mathrm{Zn}) \mathrm{Mg} \\
(\mathbf{C e}, \mathrm{Zn}) \mathrm{Mg}_{3} \\
(\mathbf{C e}, \mathrm{Zn}) \mathrm{Mg}_{12}\end{array}$ & $\begin{array}{l}\mathrm{CeMg}_{7} \mathrm{Zn}_{12} \\
\mathrm{Ce}_{2} \mathrm{Mg}_{53} \mathrm{Zn}_{45} \\
\mathrm{CeMg}_{3} \mathrm{Zn}_{5}\end{array}$ & 2010 & [39] \\
\hline $\mathrm{Mg}-\mathrm{Cu}-\mathrm{Li}$ & $\mathrm{A}$ & $\mathrm{Cu}(\mathbf{M g}, \mathrm{Li})_{2} ; \mathrm{Cu}_{2}(\mathbf{M g}, \mathrm{Li})$ & $\mathrm{Cu}_{8} \mathrm{Li}_{2} \mathrm{Mg}_{15}$ & 2000 & {$[40]$} \\
\hline $\mathrm{Mg}-\mathrm{Cu}-\mathrm{Si}$ & $\mathrm{A}$ & $\mathrm{C} 15-\mathrm{Cu}(\mathbf{M g}, \mathrm{Si})_{2}$ & $\begin{array}{l}\mathrm{Cu}_{16} \mathrm{Mg}_{6} \mathrm{Si}_{7 \_} \text {sigma } \\
\mathrm{Cu}_{3} \mathrm{Mg}_{2} \mathrm{Si} \text { _tau }\end{array}$ & 1998 & {$[41]$} \\
\hline $\mathrm{Mg}-\mathrm{Cu}-\mathrm{Y}$ & $\mathrm{A}$ & $\begin{array}{l}\text { none } \\
\mathrm{C} 14-\mathrm{Mg}(\mathrm{Cu}, \mathrm{Zn})_{2} \text {; }\end{array}$ & - & 1997 & {$[41]$} \\
\hline $\mathrm{Mg}-\mathrm{Cu}-\mathrm{Zn}$ & $\mathrm{A}$ & $\begin{array}{l}\mathrm{C} 15-\mathrm{Mg}(\mathbf{C u}, \mathrm{Zn})_{2} \\
{ }^{*} \mathrm{C} 36-\mathrm{Mg}(\mathrm{Cu}, \mathrm{Zn})_{2} \\
\mathrm{Mg}(\mathrm{Cu}, \mathbf{Z n})\end{array}$ & - & 1998 & {$[42]$} \\
\hline Mg-Gd-Li & $\mathrm{A}$ & $\begin{array}{l}(\mathbf{G d}, \mathrm{Li}) \mathrm{Mg} ;(\mathbf{G d}, \mathrm{Li}) \mathrm{Mg}_{2} ; \\
(\mathbf{G d}, \mathrm{Li}) \mathrm{Mg}_{3} ;(\mathbf{G d}, \mathrm{Li}) \mathrm{Mg}_{5}\end{array}$ & - & 2001 & [43] \\
\hline Mg-Gd-Y & B & $\begin{array}{l}(\mathbf{G d}, \mathrm{Y}) \mathrm{Mg} ;(\mathbf{G d}, \mathrm{Y}) \mathrm{Mg}_{2} \\
(\mathbf{G d}, \mathrm{Y}) \mathrm{Mg}_{3} ;(\mathbf{G d}, \mathrm{Y}) \mathrm{Mg}_{5} \\
(\mathbf{Y}, \mathrm{Gd}) \mathrm{Mg}_{2} ;(\mathbf{Y}, \mathrm{Gd})_{5} \mathrm{Mg}_{24}\end{array}$ & - & 2010 & [38] \\
\hline $\mathrm{Mg}-\mathrm{Gd}-\mathrm{Zn}$ & $\mathrm{A}$ & $\begin{array}{l}(\mathrm{Gd}, \mathrm{Zn}) \mathrm{Mg} ;(\mathrm{Gd}, \mathrm{Zn}) \mathrm{Mg}_{2} \\
(\mathbf{G d}, \mathrm{Zn}) \mathrm{Mg}_{3} ;(\mathbf{G d}, \mathrm{Zn}) \mathrm{Mg}_{5}\end{array}$ & $\begin{array}{l}14 \mathrm{H}-\mathrm{GdMg}_{12} \mathrm{Zn} \\
\text { I-Gd } \mathrm{Gg}_{68} \mathrm{Zn}_{56}\end{array}$ & 2012 & [44] \\
\hline $\mathrm{Mg}-\mathrm{Fe}-\mathrm{Si}$ & B & $\mathrm{Fe}(\mathrm{Mg}, \mathbf{S i})$ & - & 2007 & {$[45]$} \\
\hline Mg-La-Nd & $\mathrm{A}$ & $\begin{array}{l}(\mathrm{La}, \mathrm{Nd}) \mathrm{Mg} ;(\mathrm{La}, \mathrm{Nd}) \mathrm{Mg}_{3} \\
(\mathrm{La}, \mathbf{N d})_{5} \mathrm{Mg}_{41} ;(\mathbf{L a}, \mathrm{Nd}) \mathrm{Mg}_{12} \\
(\mathbf{L a}, \mathrm{Nd})_{2} \mathrm{Mg}_{17}\end{array}$ & - & 2012 & [35] \\
\hline Mg-La-Si & A & $\begin{array}{l}(\mathbf{L a}, \mathrm{Mg})_{5} \mathrm{Si}_{4} \\
(\mathbf{L a}, \mathrm{Mg})_{3} \mathrm{Si}_{2} \\
*(\mathrm{La}, \mathrm{Mg})_{0.6} \mathrm{Si}_{0.4}\end{array}$ & $\begin{array}{l}\mathrm{La}_{2} \mathrm{Mg}_{4} \mathrm{Si}_{4} \\
\mathrm{La}_{25} \mathrm{Mg}_{25} \mathrm{Si}_{50} \\
\mathrm{La}_{2} \mathrm{Mg}_{77} \mathrm{Si} \\
\mathrm{La}_{32} \mathrm{Mg}_{66} \mathrm{Si}\end{array}$ & 2010 & [46] \\
\hline
\end{tabular}


Table 2. Cont.

\begin{tabular}{|c|c|c|c|c|c|}
\hline System & $\begin{array}{l}\text { Modeling } \\
\text { status }\end{array}$ & $\begin{array}{l}\text { Ternary intermetallic } \\
\text { solid solution phases }\end{array}$ & $\begin{array}{l}\text { Ternary } \\
\text { stoichiometric phases }\end{array}$ & $\begin{array}{l}\text { Year of } \\
\text { study }\end{array}$ & Ref \\
\hline Mg-La-Zn & B & $\begin{array}{l}\mathrm{La}(\mathrm{Mg}, \mathrm{Zn}) \\
\mathrm{La}(\mathrm{Mg}, \mathrm{Zn})_{3} \\
\mathrm{La}_{2}(\mathrm{Mg}, \mathrm{Zn})_{17}\end{array}$ & I-LaMgZn & 2010 & {$[47]$} \\
\hline Mg-Li-Si & A & $(\mathrm{Mg}, \mathrm{Li})_{2} \mathrm{Si}$ & $\begin{array}{l}\mathrm{Li1}_{2} \mathrm{Mg}_{3} \mathrm{Si}_{4} \\
\mathrm{Li}_{2} \mathrm{MgSi} \\
\mathrm{Li}_{8} \mathrm{MgSi}_{6}\end{array}$ & 2004 & [48] \\
\hline $\mathrm{Mg}-\mathrm{Mn}-\mathrm{Sc}$ & B & none & - & 1999 & [49] \\
\hline $\mathrm{Mg}-\mathrm{Mn}-\mathrm{Zn}$ & B & $\begin{array}{l}\text { none } \\
(\mathrm{Y}, \mathrm{Nd}) \mathrm{Mg} ;(\mathrm{Y}, \mathbf{N d}) \mathrm{Mg}_{3}\end{array}$ & - & 2006 & {$[50]$} \\
\hline $\mathrm{Mg}-\mathrm{Nd}-\mathrm{Y}$ & B & $\begin{array}{l}(\mathrm{Y}, \mathrm{Nd})_{5} \mathrm{Mg}_{41} ;(\mathbf{Y}, \mathrm{Nd}) \mathrm{Mg}_{2} \\
(\mathbf{Y}, \mathrm{Nd})_{5} \mathrm{Mg}_{24} ; *(\mathrm{Y}, \mathrm{Nd}) \mathrm{Mg}_{5}\end{array}$ & - & 2008 & {$[51]$} \\
\hline $\mathrm{Mg}-\mathrm{Nd}-\mathrm{Zn}$ & B & $\begin{array}{l}\mathrm{Nd}(\mathrm{Mg}, \mathrm{Zn}) ; \mathrm{Nd}(\mathbf{M g}, \mathrm{Zn})_{3} \\
{ }^{*} \mathrm{Nd}_{8}(\mathrm{Mg}, \mathrm{Zn})_{92}\end{array}$ & $\begin{array}{l}\mathrm{Mg}_{35} \mathrm{Nd}_{5} \mathrm{Zn}_{60} \\
\mathrm{Mg}_{30} \mathrm{Nd}_{15} \mathrm{Zn}_{55}\end{array}$ & 2011 & {$[52]$} \\
\hline $\mathrm{Mg}-\mathrm{Si}-\mathrm{Sn}$ & A & $\mathrm{Mg}_{2}(\mathrm{Sn}, \mathrm{Si})$ & - & 2011 & {$[32]$} \\
\hline Mg-Y-Zn & A & $\begin{array}{l}\mathrm{Y}(\mathrm{Mg}, \mathrm{Zn}) ; \mathrm{Y}(\mathrm{Mg}, \mathrm{Zn})_{5} \\
* \mathrm{I}-\mathrm{YMg} \mathrm{Mg}_{3}(\mathrm{Mg}, \mathrm{Zn})_{6} \\
* \mathrm{Z}-\mathrm{Y}_{7} \mathrm{Mg}_{28}(\mathrm{Mg}, \mathrm{Zn})_{65} \\
* \mathrm{~W}-\mathrm{Y}_{25} \mathrm{Mg}_{25}(\mathrm{Mg}, \mathrm{Zn})_{50}\end{array}$ & $\begin{array}{l}14 \mathrm{H}-\mathrm{YMg}_{12} \mathrm{Zn} \\
18 \mathrm{R}-\mathrm{YMg}_{10} \mathrm{Zn}\end{array}$ & 2012 & {$[53]$} \\
\hline
\end{tabular}

* indicates a true ternary solution phase, which is the same meaning in the Table 3 .

Table 3. Ternary systems - not containing $\mathrm{Mg}$ - with complete thermodynamic descriptions and their classified modeling status. Indication details are the same as in Table 2.

\begin{tabular}{|c|c|c|c|c|c|}
\hline System & $\begin{array}{l}\text { Modeling } \\
\text { status }\end{array}$ & $\begin{array}{l}\text { Ternary intermetallic } \\
\text { solid solution phases }\end{array}$ & $\begin{array}{l}\text { Ternary } \\
\text { stoichiometric phases }\end{array}$ & $\begin{array}{l}\text { Year of } \\
\text { study }\end{array}$ & Ref. \\
\hline $\mathrm{Ag}-\mathrm{Al}-\mathrm{Cu}$ & B & none & & 2004 & [54] \\
\hline Al-C-Si & B & $(\mathrm{Al}, \mathrm{Si})_{4} \mathrm{C}_{3}$ & $\begin{array}{l}\mathrm{Al}_{4} \mathrm{SiC}_{4} \\
\mathrm{Al}_{8} \mathrm{SiC}_{7}\end{array}$ & & [55] \\
\hline $\mathrm{Al}-\mathrm{Ca}-\mathrm{Fe}$ & B & none & - & 1994 & [56] \\
\hline & & $\begin{array}{l}\mathrm{C} 15-(\mathbf{C a}, \mathrm{Sr}) \mathrm{Mg}_{2} \\
\mathrm{Al}_{4}(\mathbf{C a}, \mathrm{Sr})\end{array}$ & & & \\
\hline $\mathrm{Al}-\mathrm{Ca}-\mathrm{Sr}$ & A & $\begin{array}{l}\mathrm{Al}_{4}(\mathrm{Ca}, \mathrm{Sr}) \\
\mathrm{Al}_{2}(\mathrm{Ca}, \mathrm{Sr}) \\
\mathrm{Al}_{7}(\mathrm{Ca}, \mathrm{Sr})_{8}\end{array}$ & - & 2009 & [33] \\
\hline Al-Ce-Nd & B & $\begin{array}{l}(\mathrm{Ce}, \mathrm{Nd})_{3} \mathrm{Al}_{11} \\
(\mathrm{Ce}, \mathrm{Nd}) \mathrm{Al}_{3} \\
(\mathrm{Ce}, \mathrm{Nd}) \mathrm{Al} \\
(\mathrm{Ce}, \mathrm{Nd}) \mathrm{Al}_{2} \\
(\mathrm{Ce}, \mathrm{Nd})_{3} \mathrm{Al} \\
(\mathbf{C e}, \mathrm{Nd})_{3} \mathrm{Al}\end{array}$ & - & 2003 & [57] \\
\hline $\mathrm{Al}-\mathrm{Ce}-\mathrm{Si}$ & A & $\mathrm{Ce}(\mathrm{Al}, \mathrm{Si})_{2}$ & $\begin{array}{l}\mathrm{AlCeSi}_{2} \\
\mathrm{All}_{.6} \mathrm{CeSi}_{0.4}\end{array}$ & 2004 & [58] \\
\hline
\end{tabular}


Table 3. Cont.

\begin{tabular}{|c|c|c|c|c|c|}
\hline System & $\begin{array}{l}\text { Modeling } \\
\text { status }\end{array}$ & $\begin{array}{l}\text { Ternary intermetallic } \\
\text { solid solution phases }\end{array}$ & $\begin{array}{l}\text { Ternary } \\
\text { stoichiometric phases }\end{array}$ & $\begin{array}{l}\text { Year of } \\
\text { study }\end{array}$ & Ref. \\
\hline $\mathrm{Al}-\mathrm{Cu}-\mathrm{Gd}$ & B & $\begin{array}{l}\mathrm{Gd}(\mathrm{Al}, \mathbf{C u})_{2} \\
\mathrm{Gd}(\mathrm{Al}, \mathbf{C u})_{5} \\
\mathrm{Gd}(\mathbf{A l}, \mathrm{Cu})_{2}\end{array}$ & $\begin{array}{l}\mathrm{AlCu}_{17} \mathrm{Gd}_{2} \\
\mathrm{AlCuGd} \\
\mathrm{Al}_{2.1} \mathrm{Cu}_{0.9} \mathrm{Gd} \mathrm{Al}_{3.2} \mathrm{Cu}_{7.8} \mathrm{Gd} \\
\mathrm{Al}_{4.4} \mathrm{Cu}_{6.6} \mathrm{Gd} \\
\mathrm{Al}_{8.9} \mathrm{Cu}_{2.1} \mathrm{Gd}_{3} \\
\mathrm{Al}_{8} \mathrm{Cu}_{4} \mathrm{Gd}\end{array}$ & 2009 & [59] \\
\hline $\mathrm{Al}-\mathrm{Cu}-\mathrm{Li}$ & B & - & $\begin{array}{l}\mathrm{Al}_{2} \mathrm{CuLi} \\
\mathrm{Al}_{55} \mathrm{Cu}_{11} \mathrm{Li}_{33} \\
\mathrm{Al}_{57} \mathrm{Cu}_{11} \mathrm{Li}_{32} \\
\mathrm{Al}_{60} \mathrm{Cu}_{32} \mathrm{Li}_{8}\end{array}$ & 1994 & [41] \\
\hline $\mathrm{Al}-\mathrm{Cu}-\mathrm{Mn}$ & $\mathrm{B}$ & none & - & 2003 & {$[60]$} \\
\hline $\mathrm{Al}-\mathrm{Cu}-\mathrm{Si}$ & $\mathrm{B}$ & none & - & 1997 & [41] \\
\hline $\mathrm{Al}-\mathrm{Cu}-\mathrm{Sn}$ & $\mathrm{A}$ & none & - & 2008 & [61] \\
\hline $\mathrm{Al}-\mathrm{Cu}-\mathrm{Zn}$ & B & $(\mathrm{Al}, \mathrm{Cu})_{5} \mathrm{Cu}_{4} \mathrm{Zn}$ & - & - & [41] \\
\hline Al-Fe-Mn & B & none & - & 1997 & [41] \\
\hline $\mathrm{Al}-\mathrm{Fe}-\mathrm{Si}$ & B & - & $\begin{array}{l}\mathrm{Al}_{35} \mathrm{Fe}_{37} \mathrm{Si}_{28} \mathrm{Al}_{40} \mathrm{Fe}_{25} \mathrm{Si}_{35} \\
\mathrm{Al}_{49} \mathrm{Fe}_{16} \mathrm{Si}_{35} \\
\mathrm{Al}_{54} \mathrm{Fe}_{26} \mathrm{Si}_{20} \\
\mathrm{Al}_{60} \mathrm{Fe}_{15} \mathrm{Si}_{25} \mathrm{Al}_{64} \mathrm{Fe}_{20} \mathrm{Si}_{16} \\
\mathrm{Al}_{66} \mathrm{Fe}_{19} \mathrm{Si}_{15}\end{array}$ & 1999 & {$[62]$} \\
\hline Al-Li-Si & A & - & $\begin{array}{l}\mathrm{LiAlSi} \\
\mathrm{Li}_{5.3} \mathrm{Al}_{0.7} \mathrm{Si}_{2} \\
\mathrm{Li}_{8} \mathrm{Al}_{3} \mathrm{Si}_{5}\end{array}$ & 2001 & $\begin{array}{l}{[63]} \\
{[64]}\end{array}$ \\
\hline Al-Mn-Si & B & $\begin{array}{l}* \mathrm{Al}_{18} \mathrm{Mn}_{4}(\mathrm{Al}, \mathrm{Si}) \\
{ }^{*} \mathrm{Al}_{19} \mathrm{Mn}_{6}(\mathrm{Al}, \mathrm{Si})\end{array}$ & $\mathrm{Al}_{2} \mathrm{MnSi}_{3}$ & 1998 & [41] \\
\hline Al-Si-Zn & $\mathrm{B}$ & none & - & 1998 & {$[41]$} \\
\hline Al-Sn-Zn & $\mathrm{B}$ & none & - & 1991 & [41] \\
\hline $\mathrm{Ca}-\mathrm{Fe}-\mathrm{Si}$ & B & none & $\begin{array}{l}- \\
\mathrm{Ca}_{2} \mathrm{Li}_{5} \mathrm{Si}_{3} \\
\mathrm{Ca}_{2} \mathrm{LiSi}\end{array}$ & 1994 & {$[56]$} \\
\hline Ca-Li-Si & B & - & $\begin{array}{l}\mathrm{Ca}_{2} \mathrm{LiSi}_{3} \\
\mathrm{CaLi}_{2} \mathrm{Si} \\
\mathrm{CaLiSi}_{2}\end{array}$ & 2003 & Unpublished \\
\hline $\mathrm{Ca}-\mathrm{Sr}-\mathrm{Zn}$ & B & $\begin{array}{l}(\mathrm{Ca}, \mathrm{Sr}) \mathrm{Zn}_{2} \\
(\mathrm{Ca}, \mathrm{Sr}) \mathrm{Zn}_{5} \\
(\mathrm{Ca}, \mathrm{Sr}) \mathrm{Zn}_{13}\end{array}$ & - & 2003 & {$[65]$} \\
\hline $\mathrm{Cu}-\mathrm{Fe}-\mathrm{Si}$ & B & $\begin{array}{l}\text { none } \\
\mathrm{La}(\mathbf{C u}, \mathrm{Ni})\end{array}$ & - & 2002 & {$[66]$} \\
\hline $\mathrm{Cu}-\mathrm{La}-\mathrm{Ni}$ & B & $\begin{array}{l}\mathrm{La}(\mathbf{C u}, \mathrm{Ni})_{2} \\
\mathrm{La}(\mathbf{C u}, \mathrm{Ni})_{5} \\
\mathrm{La}(\mathrm{Cu}, \mathbf{N i})\end{array}$ & - & 2012 & {$[67]$} \\
\hline $\mathrm{Cu}-\mathrm{Sn}-\mathrm{Zn}$ & B & $\begin{array}{l}\text { none } \\
(\mathrm{Fe}, \mathrm{Mn}) \mathrm{Si}\end{array}$ & - & 1998 & {$[68]$} \\
\hline $\mathrm{Fe}-\mathrm{Mn}-\mathrm{Si}$ & B & $\begin{array}{l}(\mathrm{Fe}, \mathrm{Mn})_{5} \mathrm{Si}_{3} \\
(\mathrm{Fe}, \mathrm{Mn})_{3} \mathrm{Si}\end{array}$ & - & 1993 & [69] \\
\hline $\mathrm{Mn}-\mathrm{Y}-\mathrm{Zr}$ & $\mathrm{B}$ & none & - & 1997 & {$[70]$} \\
\hline
\end{tabular}


The large number of ternary solid phases with significant solution ranges compiled in Tables 2 and 3 highlights the necessity to go beyond simple stoichiometric binary and ternary phase descriptions. A simplified notation is used in Tables 2 and 3 to emphasize more clearly the three possibilities for ternary solid solution phases:

(i) Those extending from binary intermetallic phases with limited, though significant, solubility of the third component of the majority component are marked in a bold font. For example, the phase denoted as $\mathrm{Ag}(\mathrm{Al}, \mathrm{Mg})_{3}$ extends from the binary $\mathrm{AgMg}_{3}$ into the ternary $\mathrm{Mg}-\mathrm{Ag}$ - $\mathrm{Al}$ systems but not throughout since there is no stable $\mathrm{AgAl}_{3}$ phase.

(ii) Complete solid solubility exists, such as in the Mg-Ca-Li system between the C14-type phases $\mathrm{CaMg}_{2}$ and $\mathrm{CaLi}_{2}$. Thus, none of the two mutually substituting components $\mathrm{Mg}$ and $\mathrm{Li}$ is denoted as majority species in the $\mathrm{C} 14-\mathrm{Ca}(\mathrm{Mg}, \mathrm{Li})_{2}$ phase.

(iii) Truly ternary phases, marked by an asterisk (*), are stable over a significant solid solution range in the ternary system only, which does not extend to a binary edge system. All the phases compiled in the column "Ternary stoichiometric phases" are, of course, also truly ternary phases.

The benefit of the simplified notation presented is understood by comparison with the sublattice models actually used in the thermodynamic descriptions. For an example of case (iii) in the ternary Mg-Al-Ca system, the hexagonal ternary Laves phase C36 is modeled with a complex three-sublattice model following the crystallographic prototype for this phase in the $\mathrm{MgNi}_{2}$ structure (Pearson symbol $h P 24$, space group $\mathrm{P}_{3} / \mathrm{mmc}$ ). The stoichiometric formula used in this model is $(\mathrm{Al})_{36}(\mathrm{Al}, \mathrm{Mg})_{14}(\mathrm{Ca}, \mathrm{Mg})_{25}$, where the major species are also highlighted by bold font [18]. In Table 2 the simplified notation $* \mathrm{C} 36-\mathrm{Ca}(\mathrm{A} 1, \mathrm{Mg})_{2}$ is used for this phase in order to show at a glance that the approximate stability range occurs around 33 at.\% $\mathrm{Ca}$ in the ternary system, but not throughout to any binary edge system, as indicated by the asterisk (*). All other more complex sublattice models and mutual phase equilibria have been digested in the same manner to produce concise information on the stable solid solution ranges and to convey this message in Table 2 more clearly. For an example of case (i) in the ternary Mg-Al-Li system, the phase denoted as $\mathrm{Li}(\mathrm{Al}, \mathrm{Mg})$ clearly indicates that the binary "LiAl" phase extends with significant, though limited, solubility of $\mathrm{Mg}$ as stable phase into the ternary system. This information is not easily deduced from the actual sublattice model of this NaTl-structure-type phase including vacancies, ( $\mathrm{Li}, \mathrm{Mg}, \mathrm{Va})(\mathrm{Al}, \mathrm{Li}, \mathrm{Mg})$, which also provides the stoichiometry deviation of "LiAl" in the binary Al-Li system [22].

In addition, 23 thermodynamic descriptions for ternary systems have been developed and are implemented in the $\mathrm{Mg}$ database, classified with status $\mathrm{B}$ and unpublished. These are $8 \mathrm{Mg}$-systems: Mg-Al-Y, Mg-Cu-Ni, Mg-Gd-Mn, Mg-Li-Zn, Mg-Mn-Y, Mg-Mn-Zr, Mg-Ni-Si, Mg-Y-Zr; and 15 non-Mg systems: Al-Ca-Ce, Al-Ca-Li, Al-Ca-Si, Al-Ce-Gd, Al-Ce-La, Al-Ce-Y, Al-Cu-Nd, Al-Gd-La, Al-Gd-Nd, Al-Gd-Y, Al-La-Nd, Al-La-Y, Al-Li-Mn, Al-Mn-Sc, Cu-Ni-Si.

The importance of ternary non-Mg systems is analogous to the binary non-Mg system explained for the example of the compound $\mathrm{SiC}$ in the previous section. This is not only relevant for the secondary phases found in multicomponent $\mathrm{Mg}$ alloys, but also for applications that require calculations over a wide composition range, such as joining dissimilar materials, interface reactions between distinct alloys or materials compatibility of distinct alloys, and so on. For any thermodynamic calculation applied to multicomponent alloys it is thus useful to check if the major alloying components are 
covered by the 122 ternary systems modeled in the database (status A or B) to ensure that the calculation results are validated by underlying experimental data. For some multicomponent $\mathrm{Mg}$ alloy systems, the thermodynamic descriptions have been validated by experimental investigations. Those with a published reference are summarized in Table 4 .

The essential work to be performed for multicomponent intermetallic solution phases requires joining of the binary or ternary sublattice models into a unique and consistent description. Quaternary interaction parameters have not been used in any solution phase. The entry "none" in Table 4 means that from the cited experimental validation, using key samples, no quaternary solubility was found. Truly multicomponent phases occur very rarely. In the database, only two stoichiometric phases are modeled: $\mathrm{Al}_{5} \mathrm{Cu}_{2} \mathrm{Mg}_{8} \mathrm{Si}_{6}$ and $\mathrm{Al}_{8} \mathrm{FeMg}_{3} \mathrm{Si}_{6}$.

In fact, the solution phases given in Table 4 all originate from the binary intermetallic phase: from $\mathrm{CaMg}_{2}$ into the $\mathrm{Mg}-\mathrm{Al}-\mathrm{Ca}-\mathrm{Sr}$ and $\mathrm{Mg}-\mathrm{Al}-\mathrm{Ca}-\mathrm{Mn}-\mathrm{Sr}$ systems, and from $\mathrm{Ca}_{2} \mathrm{Sn}$ into the $\mathrm{Mg}-\mathrm{Ca}-\mathrm{Ce}-\mathrm{Sn}$ and $\mathrm{Mg}-\mathrm{Ca}-\mathrm{Si}-\mathrm{Sn}$ systems. This comprehensive and consistent solution phase modeling was also done for the intermetallic REMg and REZN phases which enables a reasonable extrapolation into multicomponent Mg-Zn-RE/Y systems $(\mathrm{RE}=\mathrm{Ce}, \mathrm{La}, \mathrm{Nd}, \mathrm{Gd})$.

Table 4. Multicomponent Mg-containing systems with verified thermodynamic descriptions and their classified modeling status. Indication details same as in Table 2.

\begin{tabular}{llllll}
\hline System & $\begin{array}{l}\text { Modeling } \\
\text { status }\end{array}$ & $\begin{array}{l}\text { Higher intermetallic } \\
\text { solid solution phases }\end{array}$ & $\begin{array}{l}\text { Higher stoichiometric } \\
\text { phases }\end{array}$ & $\begin{array}{l}\text { Year of } \\
\text { study }\end{array}$ & Ref. \\
\hline $\mathrm{Mg}-\mathrm{Al}-\mathrm{Ca}-\mathrm{Sr}$ & $\mathrm{A}$ & $\mathrm{C} 14-(\mathrm{Ca}, \mathrm{Sr})(\mathrm{Mg}, \mathrm{Al})_{2}$ & - & 2009 & {$[71]$} \\
$\mathrm{Mg}-\mathrm{Al}-\mathrm{Ca}-\mathrm{Mn}-\mathrm{Sr}$ & $\mathrm{A}$ & $\mathrm{C} 14-(\mathrm{Ca}, \mathrm{Sr})(\mathrm{Mg}, \mathrm{Al})_{2}$ & - & 2009 & {$[71]$} \\
$\mathrm{Mg}-\mathrm{Al}-\mathrm{Cu}-\mathrm{Si}$ & $\mathrm{B}$ & none & $\mathrm{Al}_{5} \mathrm{Cu}_{2} \mathrm{Mg}_{8} \mathrm{Si}_{6}$ & 2012 & {$[72]$} \\
$\mathrm{Mg}-\mathrm{Al}-\mathrm{Li}-\mathrm{Si}$ & $\mathrm{A}$ & none & - & 2001 & {$[22]$} \\
$\mathrm{Mg}-\mathrm{Al}-\mathrm{Mn}-\mathrm{Zn}$ & $\mathrm{A}$ & none & - & 2006 & {$[73]$} \\
$\mathrm{Mg}-\mathrm{Ca}-\mathrm{Ce}-\mathrm{Sn}$ & $\mathrm{A}$ & $(\mathrm{Ca}, \mathrm{Mg})(\mathrm{Ca}, \mathrm{Ce}) \mathrm{Sn}$ & - & 2012 & {$[37]$} \\
$\mathrm{Mg}-\mathrm{Ca}-\mathrm{Si}-\mathrm{Sn}$ & $\mathrm{A}$ & $(\mathrm{Ca}, \mathrm{Mg}) \mathrm{Ca}(\mathrm{Sn}, \mathrm{Si})$ & - & 2011 & {$[32]$} \\
$\mathrm{Mg}-\mathrm{Ce}-\mathrm{Gd}-\mathrm{Y}$ & $\mathrm{B}$ & none & - & 2010 & {$[38]$} \\
$\mathrm{Mg}-\mathrm{Ce}-\mathrm{Mn}-\mathrm{Sc}$ & $\mathrm{B}$ & none & - & 2001 & {$[74]$} \\
$\mathrm{Mg}-\mathrm{Gd}-\mathrm{Mn}-\mathrm{Sc}$ & $\mathrm{B}$ & none & - & 2001 & {$[74]$} \\
$\mathrm{Mg}-\mathrm{Mn}-\mathrm{Sc}-\mathrm{Y}$ & $\mathrm{B}$ & none & - & 2001 & {$[74]$} \\
\hline
\end{tabular}

At this point, the rule of thumb is reflected and evidenced: The more components included, the less additional information is required. This rule is proper for $\mathrm{Mg}$ alloy thermodynamics, on the basis of meticulous Calphad modeling of the binary and ternary systems and consistent treatment of multicomponent intermetallic solution phases.

\section{Crystal Structure Guided Modeling of Multicomponent Intermetallic Solution Phases}

In the previous chapter, the experimentally validated stability ranges of ternary intermetallic solution phases in the individual ternary and some multicomponent systems have been worked out. The focus of this chapter is on the proper modeling of such phases considering the information given by the crystal structure. The basic idea is that intermetallic phases occurring in different binary systems may be modeled as the same phase if they share the same crystal structure. If, in addition, the lattice 
parameters are similar and the components do not exhibit strong repulsive interactions, the simplest realistic description is that of a single phase with ideal substitutional solution. That is certainly a better approximation compared to the implementation as separate compound phases, which is in fact equivalent to complete demixing.

This approach is detailed in the following examples, highlighting the diversity of such unified phases. The continuous solubility or the solubility limits of such phases eventually result from the competition of phases as modeled in the database.

One example depicted in Figure 3 is the phase "REMg", emerging from ten stable binary REMg or REZn phases with the common $c P 2-\mathrm{CsCl}$ crystal structure. Accordingly, it is modeled with two sublattices with site ratios 1:1. On the first sublattice, the rare earths (Ce,Gd,La,Nd,Y) are majority components, denoted by bold font, whereas $\mathrm{Mg}$ and $\mathrm{Zn}$ are minority components denoted by an italic font. On the second sublattice $(\mathrm{Mg}, \mathrm{Zn})$ are majority and (Al,Mn,Li) minority components, respectively. The minority species cannot reach a complete occupancy on their sublattice in the stable region of the phase named REMg. Nevertheless, it is essential to assess a reasonable Gibbs energy function for all the metastable end member phases, such as the "CeAl" composition of the REMg phase. The REMg phase occurs as stable continuous solid solution (Ce, $\mathrm{Nd}) \mathrm{Mg}$ in the $\mathrm{Mg}-\mathrm{Ce}-\mathrm{Nd}$ system, Figure 4, and as $\mathrm{Ce}(\mathrm{Mg}, \mathrm{Zn})$ solution in the $\mathrm{Mg}-\mathrm{Ce}-\mathrm{Zn}$ system, Figure 5. In the Mg-Al-Ce system, it is a limited solid solution $\mathrm{Ce}(\mathrm{Mg}, \mathrm{Al})$ as shown in Figure 6. In these calculated ternary isothermal phase diagram sections the ternary intermetallic solution phases are highlighted by red lines and the ternary stoichiometric phases by red dots. Three-phase regions (triangles) are shaded.

Figure 3. Sketch of the unified model of the multicomponent phase REMg and its connection to all stable binary phases.




Figure 4. Calculated isothermal $\mathrm{Mg}-\mathrm{Ce}-\mathrm{Nd}$ phase diagram at $500{ }^{\circ} \mathrm{C}$ highlighting intermetallic phases with continuous (3) or limited (1) ternary solubility [36].

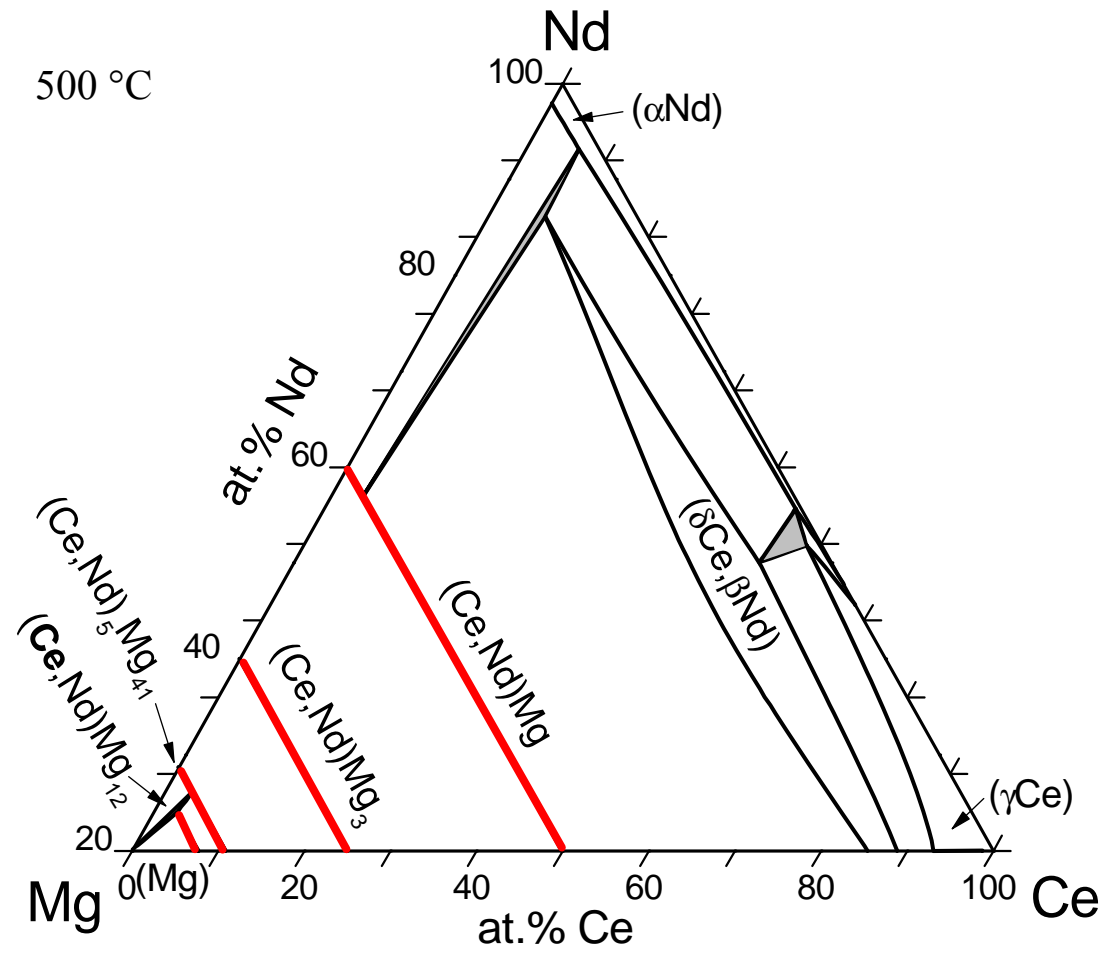

Figure 5. Calculated isothermal $\mathrm{Mg}-\mathrm{Ce}-\mathrm{Zn}$ phase diagram at $300{ }^{\circ} \mathrm{C}$ highlighting intermetallic phases with continuous (1) or limited (2) ternary solubility, and additionally three ternary stoichiometric phases [39].

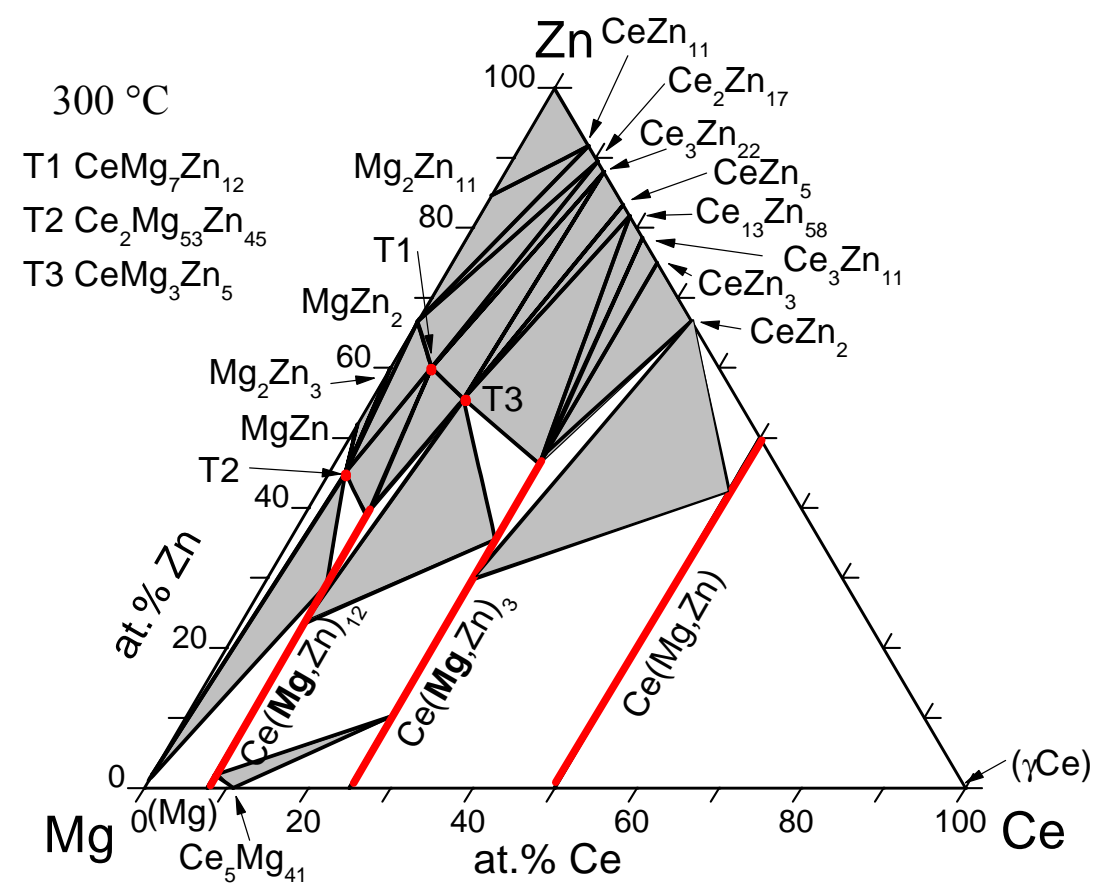

In the Mg-Ce-Nd system, Figure 4, the Mg-rich corner is "locked-in" by the continuous solid solution of the $(\mathrm{Ce}, \mathrm{Nd})_{5} \mathrm{Mg}_{41}$ phase. Therefore, only this phase is expected together with $(\mathrm{Ce}, \mathrm{Nd}) \mathrm{Mg}_{12}$ 
in Mg-rich alloys with $\mathrm{Ce}$ and $\mathrm{Nd}$. In as-cast microstructures, the (Ce, $\mathrm{Nd})_{5} \mathrm{Mg}_{41}$ phase is typically suppressed by impeded nucleation/growth. The resulting metastable phase diagram reveals a drastic extension of the primary crystallization field of $(\mathrm{Ce}, \mathrm{Nd}) \mathrm{Mg}_{12}$ and this phase is in fact found in as-cast samples [36].

The Mg-Ce-Zn system, Figure 5, looks even more complicated. Three ternary solubilities are observed, including one continuous solid solution and, additionally, three ternary stoichiometric phases. Mg-rich alloys with $\mathrm{Ce}$ and $\mathrm{Zn}$ show the secondary phase $(\mathrm{Ce}, \mathrm{Zn}) \mathrm{Mg}_{12}$ with large $\mathrm{Zn}$ content up to 40 at.\% $\mathrm{Zn}$ and possibly the ternary phase $\mathrm{T} 2, \mathrm{Ce}_{2} \mathrm{Mg}_{53} \mathrm{Zn}_{45}$ depending on the $\mathrm{Ce}: \mathrm{Zn}$ ratio [39].

Figure 6. Calculated isothermal Mg-Al-Ce phase diagram at $400{ }^{\circ} \mathrm{C}$ highlighting the single intermetallic solution phase $\mathrm{Ce}(\mathrm{Al}, \mathrm{Mg})_{2}$ which is not continuous at $400{ }^{\circ} \mathrm{C}$ but at $740{ }^{\circ} \mathrm{C}[19]$.

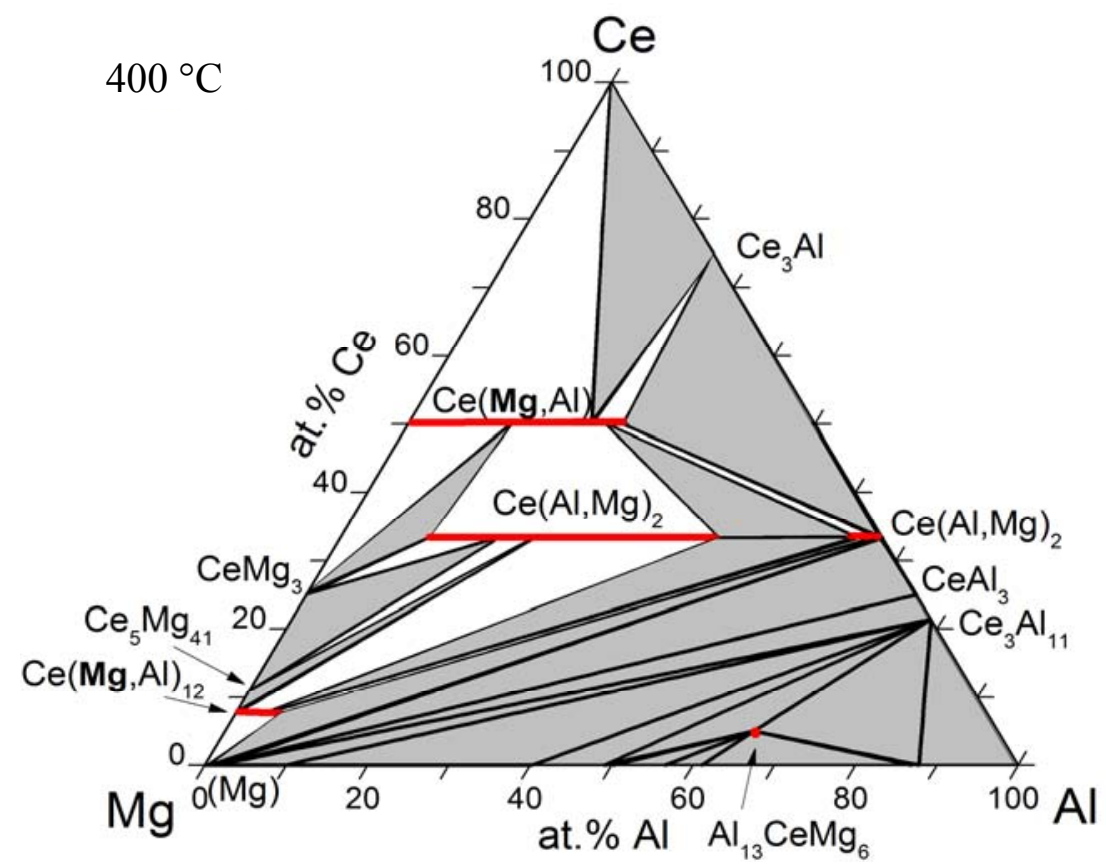

Figure 6 shows the $\mathrm{Mg}$-Al-Ce system at $400{ }^{\circ} \mathrm{C}$ with limited solubility of the third component in $\left(\mathrm{Ce}(\mathrm{Mg}, \mathrm{Al})\right.$ and $\mathrm{Ce}(\mathrm{Mg}, \mathrm{Al})_{12}$ and one ternary stoichiometric phase $\mathrm{Al}_{13} \mathrm{CeMg}_{6}$. Apparently the phase $\mathrm{Ce}(\mathrm{Al}, \mathrm{Mg})_{2}$ occurs in two separate composition ranges and might be even mistaken for a ternary phase. However, at higher temperatures, around $740{ }^{\circ} \mathrm{C}$, a complete solution range between $\mathrm{CeAl}_{2}$ and $\mathrm{CeMg}_{2}$ is established [19] (see also Table 2). For Mg-rich alloys, the $\mathrm{Al}$ solubility of the $\mathrm{Ce}(\mathrm{Mg}, \mathrm{Al})_{12}$ phase is crucial, since it blocks the way to an equilibrium of $(\mathrm{Mg})$ with the mid-composition $\mathrm{Ce}(\mathrm{Al}, \mathrm{Mg})_{2}$ phase during solidification and heat treatments.

In the Mg-Al-Sr system at $400{ }^{\circ} \mathrm{C}$, Figure 7, a similar cut-off is expected due to the Al solubility of the $\mathrm{Sr}_{2}(\mathrm{Mg}, \mathrm{Al})_{17}$ phase. In this system, even the ternary $\mathrm{Al}_{38} \mathrm{Mg}_{58} \mathrm{Sr}_{4}$ can be formed in $\mathrm{Mg}$-rich alloys during Scheil solidification [23]. 
Figure 7. Calculated isothermal $\mathrm{Mg}$-Al-Sr phase diagram at $400{ }^{\circ} \mathrm{C}$ highlighting six intermetallic solution phases with limited ternary solubility plus one ternary stoichiometric phase [26].

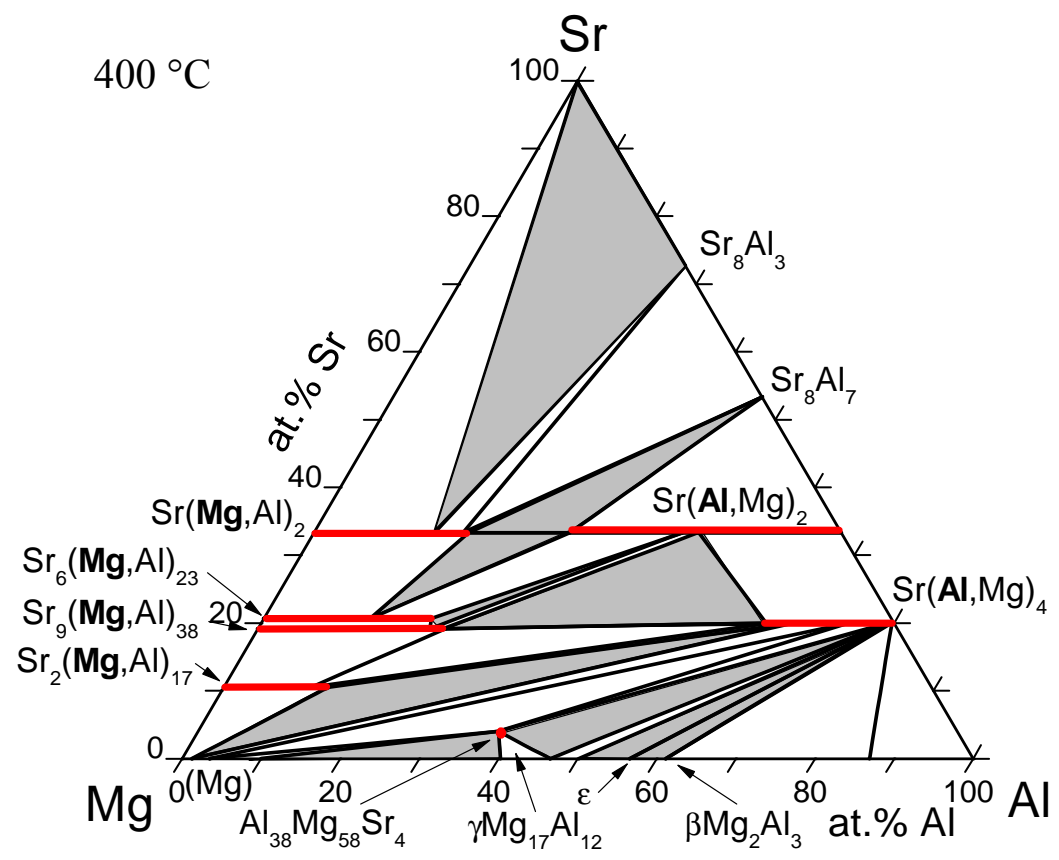

The six stable binary end members of the multicomponent phase " $R E_{2} \mathrm{Mg}_{17}$ " sketched in Figure 8 crystallize in the $h P 38-\mathrm{Ni}_{17} \mathrm{Th}_{2}$ structure type. The four $\mathrm{RE}_{2} \mathrm{Zn}_{17}$ phases are stable down to room temperature whereas $\mathrm{Ce}_{2} \mathrm{Mg}_{17}$ and $\mathrm{La}_{2} \mathrm{Mg}_{17}$ are stable at high temperature only. Therefore, $\mathrm{Ce}_{2} \mathrm{Mg}_{17}$ does not appear in the isothermal $\mathrm{Mg}-\mathrm{Ce}-\mathrm{Nd}$ section at $500{ }^{\circ} \mathrm{C}$ in Figure 4. At higher temperature, the $\mathrm{RE}_{2} \mathrm{Mg}_{17}$ phase becomes stable in this system and the limited $\mathrm{Nd}$ solubility in $\mathrm{Ce}_{2} \mathrm{Mg}_{17}$ is modeled, considering the Gibbs energy of the metastable end member $\mathrm{Nd}_{2} \mathrm{Mg}_{17}$ marked in orange in Figure 8 .

Figure 8. Sketch of the unified model of the multicomponent phase $\mathrm{RE}_{2} \mathrm{Mg}_{17}$ and its connection to all stable binary phases and the metastable end member phase $\mathrm{Nd}_{2} \mathrm{Mg}_{17}$ (orange).

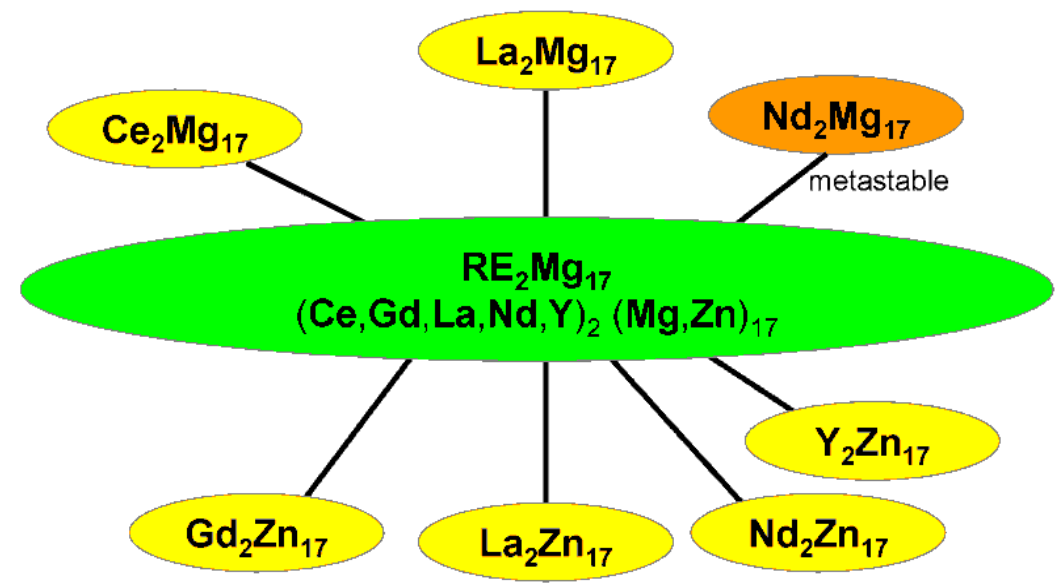


Two non-Mg systems will be given as examples for reasonably calculated phase diagrams without using any ternary parameter after unification of binary intermetallics crystallizing in the same crystal structure. Figure 9 shows the Ce-La-Si system where five binary compounds were unified to ternary continuous solid solutions, since they share identical crystal structures of the corresponding binary phases:

- $(\mathrm{Ce}, \mathrm{La}) \mathrm{Si}_{2}\left(t I 12-\mathrm{ThSi}_{2}\right.$ structure type)

- (Ce,La)Si (oP8-FeB structure type)

- $(\mathrm{Ce}, \mathrm{La})_{5} \mathrm{Si}_{4}\left(\mathrm{Zr}_{5} \mathrm{Si}_{4}\right.$ structure type)

- $(\mathrm{Ce}, \mathrm{La})_{3} \mathrm{Si}_{2}\left(t P 10-\mathrm{U}_{3} \mathrm{Si}_{2}\right.$ structure type)

- $(\mathrm{Ce}, \mathrm{La})_{5} \mathrm{Si}_{3}-\mathrm{HT}\left(t I 32-\mathrm{Cr}_{5} \mathrm{~B}_{3}\right.$ structure type)

This calculated (predicted) isothermal phase diagram in Figure 9 is confirmed by the experimental work of Bulanova et al. [75].

Figure 9. Calculated (predicted) isothermal Ce-La-Si phase diagram at $500{ }^{\circ} \mathrm{C}$ with five continuous solid solutions.

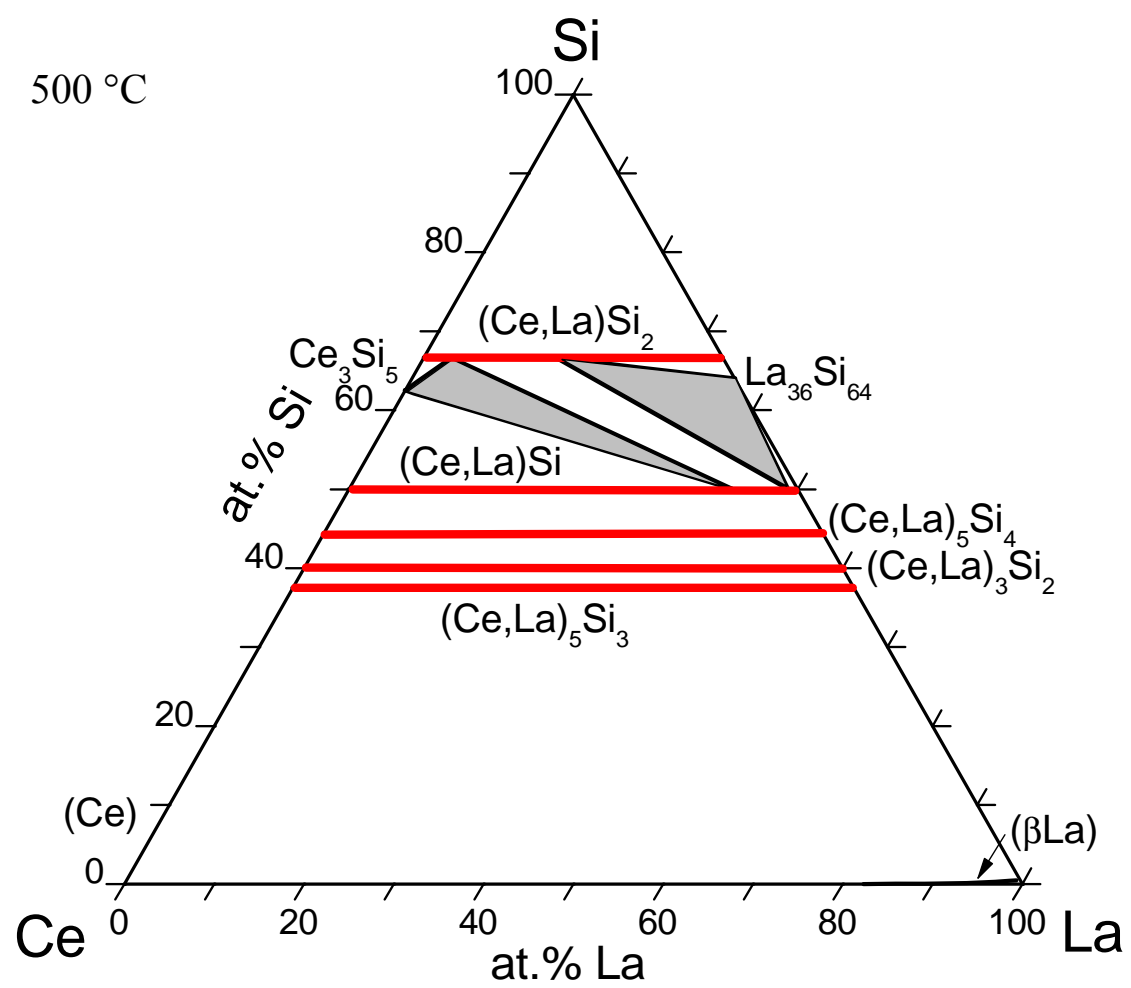

The comprehensive modeling of the phase "CaCu" $\left(h P 6-\mathrm{CaCu}_{5}\right.$ structure type), which unifies five $\mathrm{Cu}$ - and five Ni-containing phases is illustrated in Figure 10. Focusing on the $\mathrm{Ce}-\mathrm{Cu}-\mathrm{Ni}$ system, this includes the phases $\mathrm{CeCu}_{5}$ and $\mathrm{CeNi}_{5}$, sharing the $\mathrm{CaCu}_{5}$ structure type, which are therefore modeled as one phase $\mathrm{Ce}(\mathrm{Cu}, \mathrm{Ni})_{5}$. Experimental proof of this continuous solid solution of $\mathrm{Ce}(\mathrm{Cu}, \mathrm{Ni})_{5}$ was given by [76]. 
Figure 10. Sketch of the unified model of the multicomponent phase $\mathrm{CaCu}_{5}$ and its connection to all stable binary phases.

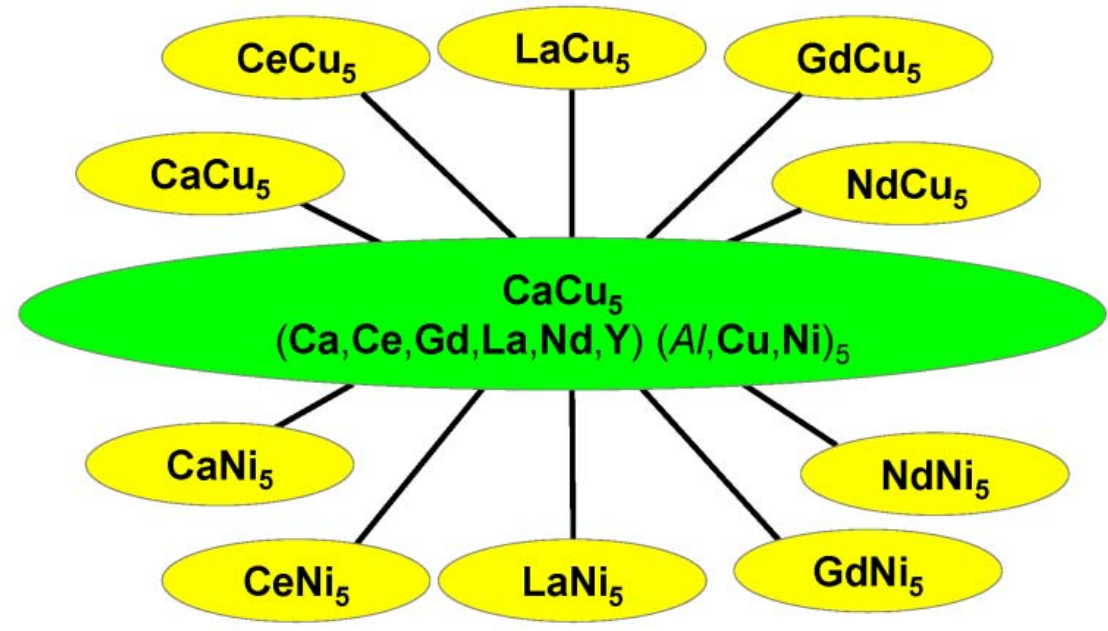

However, the phases $\mathrm{CeCu}_{2}\left(\mathrm{CeCu}_{2}\right.$-type structure) and $\mathrm{CeNi}_{2}\left(\mathrm{CuMg}_{2}\right.$-type structure $)$ crystallize in different structure types and cannot be joined. The same applies to the phases $\mathrm{CeCu}$ (FeB-type structure) and $\mathrm{CeNi}$ (CrB-type structure) which must also be modeled as separate phases. Their very limited ternary solubilities [77] are not considered in this first-stage description. Based on these considerations, the ternary $\mathrm{Ce}-\mathrm{Cu}-\mathrm{Ni}$ phase diagram in Figure 11 can be calculated without using any ternary interaction parameter as an extrapolation from the binary systems. This provides a reasonable estimation for this system, especially in Ce-poor regions.

Figure 11. Calculated isothermal $\mathrm{Ce}-\mathrm{Cu}-\mathrm{Ni}$ section at $300{ }^{\circ} \mathrm{C}$ with one continuous solid solution.

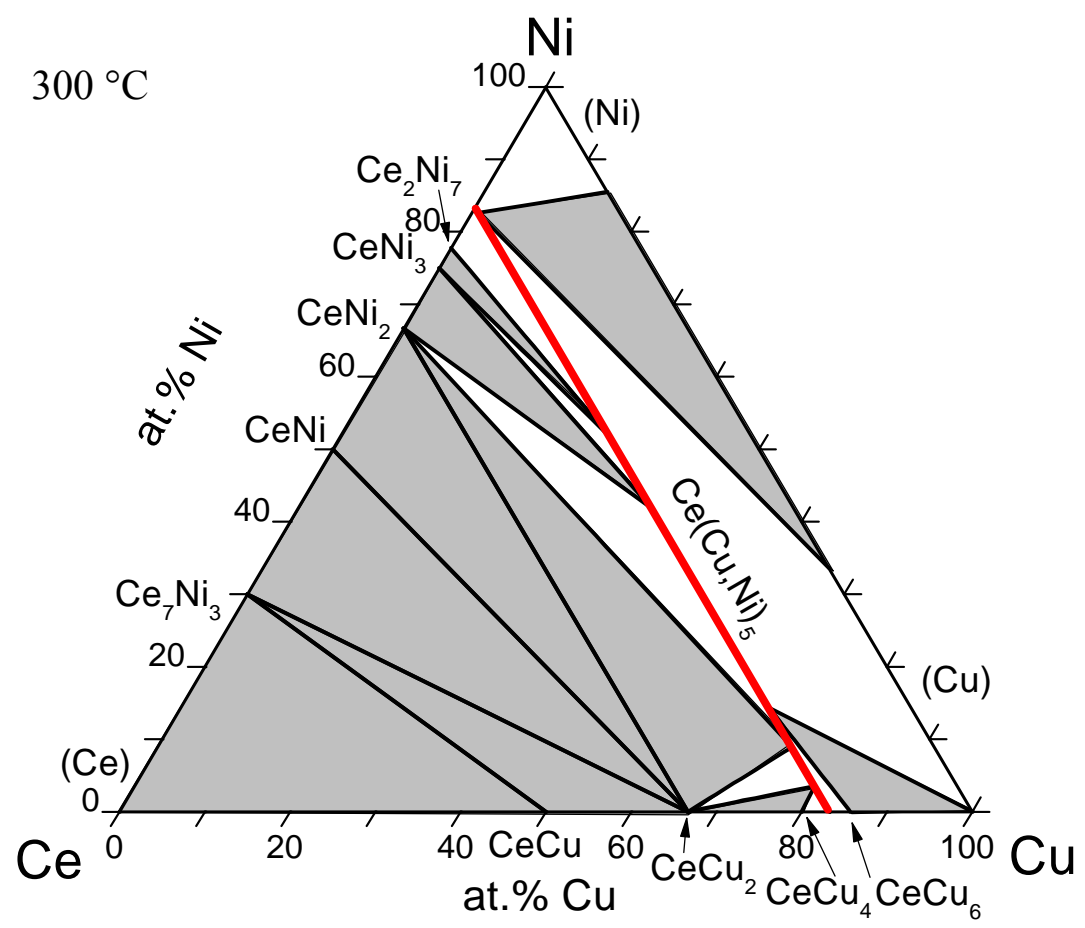




\section{Conclusions}

Intermetallic solid solution phases extending to ternary or higher alloy systems are shown to be abundant in $\mathrm{Mg}$ alloys. Therefore, calculations that are not based on proper thermodynamic descriptions of at least the important ternary systems may be precarious. Describing only the binary compounds is inadequate for ternary or higher $\mathrm{Mg}$ alloy applications.

Thermodynamic descriptions of intermetallic solution phases should be guided by their crystal structure. Generally, such phases occurring in different binary systems may be modeled as the same phase if they share the same crystal structure. Joining such phases enables more realistic predictions of multicomponent alloy phase diagrams and phase formation calculations. The diversity of such unified phases is emphasized.

On the basis of meticulous Calphad modeling of the binary and ternary systems and consistent treatment of multicomponent intermetallic solution phases, the following rule of thumb is proper for $\mathrm{Mg}$ alloy thermodynamics: The more components that are included, the less additional information is required. That enables applications of truly multicomponent $\mathrm{Mg}$ alloys involving thermodynamic calculation of any kind of phase diagram sections or liquidus and solidus projections, solidification simulation using the limiting Scheil and equilibrium conditions and obtaining thermodynamic driving forces for kinetic processes.

\section{Conflict of Interest}

The authors declare no conflict of interest.

\section{References}

1. Luo, A.A.; Fu, P.; Peng, L.; Kang, X.; Li, Z.; Zhu, T. Solidification microstructure and mechanical properties of cast magnesium-aluminum-tin alloys. Met. Mater. Trans. 2012, 43A, 360-368.

2. Luo, A.A.; Powell, B.R.; Sachdev, A.K. Computational phase equilibria and experimental investigation of magnesium-aluminum-calcium alloys. Intermetallics 2012, 24, 22-29.

3. Zhang, C.; Luo, A.A.; Chang, A. The solidification microstructure and precipitation investigation of magnesium-rich alloys containing $\mathrm{Zn}$ and Ce. In Magnesium Technology 2011; Sillekens, W.H., Agnew, S.R., Neelameggham, N.R., Mathaudhu, S.N., Eds.; The Minerals, Metals Materials Society: Warrendale, PA, USA; 2011; pp. 267-270.

4. Hänzi, A.C.; Dalla Torre, F.H.; Zhao, L.; Gunde, P.; Schmid-Fetzer, R.; Kuehlein, M.; Löffler, J.F.; Uggowitzer, P.J. Design strategy for microalloyed ultra-ductile magnesium alloys. Phil. Mag. Lett. 2009, 89, 377-390.

5. Kaufman, L.; Bernstein, H. Computer Calculations of Phase Diagrams; Academic Press: New York, NY, USA, 1970.

6. Lukas, H.L.; Fries, S.G.; Sundman, B. Computational Thermodynamics: The Calphad Method; Cambridge University Press: Cambridge, UK, 2007.

7. Pisch, A.; Schmid-Fetzer, R. Development of a thermodynamic Mg-alloy database by experiments and modeling. In Magnesium Alloys and Their Applications; Mordike, B.L., Kainer, K.U., Eds.; Wiley-VCH Verlag GmbH: Frankfurt, Germany, 1998; pp. 139-144. 
8. Schmid-Fetzer, R.; Gröbner, J. Focused development of magnesium alloys using the calphad approach. Adv. Eng. Mater. 2001, 3, 947-961.

9. Schmid-Fetzer, R.; Janz, A.; Gröbner, J.; Ohno, M. Aspects of quality assurance in a thermodynamic Mg alloy database. Adv. Eng. Mater. 2005, 7, 1142-1149.

10. Gröbner, J.; Janz, A.; Kozlov, A.; Mirkovic, D.; Schmid-Fetzer, R. Phase diagrams of advanced magnesium alloys containing Al, Ca, Sn, Sr and Mn. JOM 2008, 12, 32-38.

11. Gröbner, J.; Schmid-Fetzer, R. Key issues in thermodynamic Mg alloy database. Metall. Mater. Trans. submitted for publication, 2012.

12. Andersson, J.O.; Helander, T.; Höglund, L.; Shi, P.; Sundman, B. Thermo-Calc \& DICTRA, computational tools for materials science. Calphad 2002, 26, 273-312.

13. Bale, C.W.; Bélisle, E.; Chartrand, P.; Decterov, S.A.; Eriksson, G.; Hack, K.; Jung, I.-H.; Kang, Y.-B.; Melançon, J.; Pelton, A.D.; et al. FactSage thermochemical software and databases-Recent developments. Calphad 2009, 33, 295-311.

14. Cao, W.; Chen, S.; Zhang, F.; Wu, K.; Yang, Y.; Chang, Y.; Schmid-Fetzer, R.; Oates, W.A. PANDAT software with PanEngine, PanOptimizer and PanPrecipitation for multi-component phase diagram calculation and materials property simulation. Calphad 2009, 33, 328-342.

15. Dinsdale, A.T. SGTE data for pure elements. Calphad 1991, 15, 317-425.

16. Lim, M.S.; Tibballs, J.E.; Rossiter, P.L. An assessment of thermodynamic equilibria in the $\mathrm{Ag}-\mathrm{Al}-\mathrm{Cu}-\mathrm{Mg}$ quaternary system in relation to precipitation reactions. Z. Metallkd. 1997, 88, 236-245.

17. Gröbner, J.; Kevorkov, D.; Chumak, I.; Schmid-Fetzer, R. Experimental investigation and thermodynamic calculation of ternary Al-Ca-Mg phase equilibria. Z. Metallkde. 2003, 94, 976-982.

18. Janz, A.; Gröbner, J.; Cao, H.; Zhu, J.; Chang, Y.A.; Schmid-Fetzer, R. Thermodynamic modeling of the Mg-Al-Ca system. Acta Mater. 2009, 57, 682-694.

19. Gröbner, J.; Kevorkov, D.; Schmid-Fetzer, R. Thermodynamic modeling of Al-Ce-Mg equilibria coupled with key experiments. Intermetallics 2002, 10, 415-422.

20. Buhler, T.; Fries, S.G.; Spencer, P.J.; Lukas, H.L. A thermodynamic assessment of the Al-Cu-Mg ternary system. J. Phase Equilbria 1998, 19, 317-329.

21. Gröbner, J.; Kevorkov, D.; Schmid-Fetzer, R. Thermodynamic calculation of Al-Gd and Al-Gd-Mg phase equilibria checked by key experiments. Z. Metallkde. 2001, 92, 22-27.

22. Kevorkov, D. Thermodynamics and Phase Equilibria of the Mg-Al-Li-Si System. Ph.D dissertaion, Technische Universität Clausthal, Clausthal-Zellerfeld, Germany, 2001.

23. Du, Y.; Wang, J.; Zhao, J.; Schuster, J.C.; Weitzer, F.; Schmid-Fetzer, R.; Ohno, M.; Xu, H.; Liu, Z.K.; Shang, S.; et al. Reassessment of the Al-Mn system and a thermodynamic description of the Al-Mg-Mn system. Int. J. Mater. Res. 2007, 98, 855-871.

24. Ohno, M.; Schmid-Fetzer, R. Thermodynamic assessment of Mg-Al-Mn phase equilibria, focusing on Mg-rich alloys. Z. Metallkde. 2005, 96, 857-869.

25. Gröbner, J.; Schmid-Fetzer, R.; Pisch, A.; Cacciamani, G.; Riani, P.; Parodi, N.; Borzone, G.; Saccone, A.; Ferro, R. Experimental investigations and thermodynamic calculation in the Al-Mg-Sc system. Z. Metallkde. 1999, 90, 872-880. 
26. Janz, A.; Gröbner, J.; Mirkovic, D.; Medraj, M.; Zhu, J.; Chang, Y.A.; Schmid-Fetzer, R. Experimental study and thermodynamic calculation of $\mathrm{Al}-\mathrm{Mg}-\mathrm{Sr}$ phase equilibria. Intermetallics 2007, 15, 506-519.

27. Doernberg, E.; Kozlov, A.; Schmid-Fetzer, R. Experimental investigation and thermodynamic calculation of Mg-Al-Sn phase equilibria and solidification microstructures. J. Phase Equilbria Diffus. 2007, 28, 523-535.

28. Ohno, M.; Mirkovic, D.; Schmid-Fetzer, R. Phase equilibria and solidification of Mg-rich Mg-Al-Zn alloys. Mater. Sci. Eng. A 2006, 421, 328-337.

29. Hampl, M.; Gröbner, J.; Schmid-Fetzer, R. Experimental study of phase equilibria and solidification microstructures of $\mathrm{Mg}-\mathrm{Ca}-\mathrm{Ce}$ alloys combined with thermodynamic modelling. J. Mater. Sci. 2007, 42, 10023-10031.

30. Gröbner, J.; Schmid-Fetzer, R.; Pisch, A.; Colinet, C.; Pavlyuk, V.V.; Dmytriv, G.S.; Kevorkov, D.G.; Bodak, O.I. Phase equilibria, calorimetric study and thermodynamic modeling of Mg-Li-Ca alloys. Thermochim. Acta 2002, 389, 85-94.

31. Gröbner, J.; Chumak, I.; Schmid-Fetzer, R. Experimental study of ternary Ca-Mg-Si phase equilibria and thermodynamic assessment of $\mathrm{Ca}-\mathrm{Si}$ and $\mathrm{Ca}-\mathrm{Mg}-\mathrm{Si}$ systems. Intermetallics 2003, 11, 1065-1074.

32. Kozlov, A.; Gröbner, J.; Schmid-Fetzer, R. Phase formation in Mg-Sn-Si and Mg-Sn-Si-Ca alloys. J. Alloys Comp. 2011, 509, 3326-3337.

33. Janz, A.; Schmid-Fetzer, R. Thermodynamics and constitution of Mg-Al-Ca-Sr-Mn alloys: Part I. Experimental investigation and thermodynamic modeling of subsystems $\mathrm{Mg}-\mathrm{Ca}-\mathrm{Sr}$ and $\mathrm{Al}-\mathrm{Ca}-\mathrm{Sr}$. J. Phase Equilbria Diffus. 2009, 30, 146-156.

34. Brubaker, C.O.; Liu, Z.K. A computational thermodynamic model of the $\mathrm{Ca}-\mathrm{Mg}-\mathrm{Zn}$ system. J. Alloys Comp. 2004, 370, 114-122.

35. Gröbner, J.; Hampl, M.; Schmid-Fetzer, R.; Easton, M.A.; Zhu, S.; Gibson, M.A.; Nie, J.F. Phase analysis of Mg-La-Nd and Mg-Ce-La alloys. Intermetallics 2012, 28, 92-101.

36. Gröbner, J.; Kozlov, A.; Schmid-Fetzer, R.; Easton, M.A.; Zhu, S.; Gibson, M.A.; Nie, J.F. Thermodynamic analysis of as-cast and heat-treated microstructures of $\mathrm{Mg}-\mathrm{Ce}-\mathrm{Nd}$ alloys. Acta Mater. 2011, 59, 613-622.

37. Kozlov, A.; Gröbner, J.; Schmid-Fetzer, R. Phase formation in Mg-Sn alloys modified by Ca and Ce. J. Phase Equilbria Diffus., submitted for publication, 2012.

38. Gröbner, J.; Schmid-Fetzer, R. Thermodynamic modeling of the Mg-Ce-Gd-Y system. Scripta Mater. 2010, 63, 674-679.

39. Chiu, C.N.; Gröbner, J.; Kozlov, A.; Schmid-Fetzer, R. Experimental study and thermodynamic assessment of ternary $\mathrm{Mg}-\mathrm{Zn}$-Ce phase relations focused on Mg-rich alloys. Intermetallics 2010, 18, 399-405.

40. Braga, M.H.; Malheiros, L.F.; Haemaelaeinen, M. The Cu-Li-Mg system at room temperature. Thermochim. Acta 2000, 344, 47-54.

41. Ansara, I.; Dinsdale, A.T.; Rand, M.H. COST507-Definition of Thermochemical and Thermophysical Properties to Provide a Database for the Development of New Light Alloys, Volume 2: Thermochemical Database for Light Metal Alloys; Office for Official Publications of the European Communities: Luxembourg, Luxembourg, 1998. 
42. Liang, P.; Seifert, H.J.; Lukas, H.L.; Ghosh, G.; Effenberg, G.; Aldinger, F. Thermodynamic modelling of the $\mathrm{Cu}-\mathrm{Mg}-\mathrm{Zn}$ ternary system. Calphad 1998, 22, 527-544.

43. Kevorkov, D.G.; Gröbner, J.; Schmid-Fetzer, R.; Pavlyuk, V.V.; Dmytriv, G.S.; Bodak, O.I. The ternary Gd-Li-Mg system: Phase diagram study and computational evaluation. J. Phase Equilbria 2001, 22, 34-42.

44. Gröbner, J.; Kozlov, A.; Fang, X.; Geng, J.; Nie, J.F.; Schmid-Fetzer, R. Phase equilibria and transformations in ternary $\mathrm{Mg}$-rich $\mathrm{Mg}-\mathrm{Gd}-\mathrm{Zn}$ alloys including the thermodynamic optimization of the binary Gd-Zn. J. Alloys Comp., submitted for publication, 2012.

45. Du, Y.; Zhao, J.R.; Zhang, C.; Chen, H.L.; Zhang, L.J. Thermodynamic modeling of the Fe-Mg-Si system. J. Min. Metall. 2007, 43B, 39-56.

46. Zhou, S.; Liu, L.; Yuan, X.; Zheng, F.; Jin, Z. Thermodynamic assessment of La-Si and Mg-La-Si systems. J. Alloys Comp. 2010, 490, 253-259.

47. Qi, H.Y.; Huang, G.X.; Liu, R.D.; Zhang, K.; Liu, L.B.; Jin, Z.P. Thermodynamic optimization of La-Zn and La-Mg-Zn systems. J. Alloys Comp. 2010, 497, 336-343.

48. Kevorkov, D.; Schmid-Fetzer, R.; Zhang, F. Phase eguilibria and thermodynamics of the Mg-Si-Li system and remodeling of the Mg-Si system. J. Phase Equilbria Diffus. 2004, 25, $140-151$.

49. Von Buch, F.; Lietzau, J.; Mordike, B.L.; Pisch, A.; Schmid-Fetzer, R. Development of Mg-Sc-Mn alloys. Mater. Sci. Eng. A 1999, 263, 1-7.

50. Ohno, M.; Schmid-Fetzer, R. Mg-rich phase equilibria of Mg-Mn-Zn alloys analyzed by computational thermochemistry. Int. J. Mater. Res. 2006, 97, 526-532.

51. Guo, C.P.; Du, Z.M.; Li, C.R. Thermodynamic description of the Ce-Mg-Y and Mg-Nd-Y systems. Int. J. Mater. Res. 2008, 99, 650-668.

52. Zhang, C.; Luo, A.A.; Peng, L.M.; Stone, D.S.; Chang, Y.A. Thermodynamic modeling and experimental investigation of the magnesium-neodymium-zinc alloys. Intermetallics 2011, 19, $1720-1726$.

53. Gröbner, J.; Kozlov, A.; Fang, X.; Geng, J.; Nie, J.F.; Schmid-Fetzer, R. Phase equilibria and transformations in ternary Mg-rich Mg-Y-Zn alloys. Acta Mater., in press.

54. Witusiewicz, V.T.; Hecht, U.; Fries, S.G.; Rex, S. The Ag-Al-Cu system II. A thermodynamic evaluation of the ternary system. J. Alloys Comp. 2004, 387, 217-227.

55. Gröbner, J.; Lukas, H.L.; Aldinger, F. Thermodynamic calculation of the Al-Si-C ternary system. Calphad 1996, 20, 247-254.

56. Anglezio, J.C.; Servant, C.; Ansara, I. Contribution to the experimental and thermodynamic assessment of the Al-Ca-Fe-Si system-I. Al-Ca-Fe, Al-Ca-Si, Al-Fe-Si and Ca-Fe-Si systems. Calphad 1994, 18, 273-309.

57. Cacciamani, G.; Cardinale, A.M.; Borzone, G.; Ferro, R. Thermodynamic modelling and optimization of the Al-Ce-Nd system. Calphad 2003, 27, 227-233.

58. Gröbner, J.; Mirkovic, D.; Schmid-Fetzer, R. Thermodynamic aspects of the constitution, grain refining and solidification enthalpies of Al-Ce-Si alloys. Metall. Mater. Trans A 2004, 35, 3349-3362.

59. Zhang, L.G.; Dong, H.Q.; Huang, G.X.; Shan, J.; Liu, L.B.; Jin, Z.P. Thermodynamic assessment of the Al-Cu-Gd system. Calphad 2009, 33, 664-672. 
60. Miettinen, J. Thermodynamic description of the $\mathrm{Cu}-\mathrm{Al}-\mathrm{Mn}$ system in the copper-rich corner. Calphad 2003, 27, 103-114.

61. Mirković, D.; Gröbner, J.; Schmid-Fetzer, R. Liquid demixing and microstructure formation in ternary Al-Sn-Cu alloys. Mat. Sci. Eng. A 2008, 487, 456-467.

62. Liu, Z.K.; Chang, Y.A. Thermodynamic assessment of the Al-Fe-Si system. Metall. Mater. Trans A 1999, 30A, 1081-1095.

63. Gröbner, J.; Kevorkov, D.; Schmid-Fetzer, R. The Al-Li-Si system, Part 2: Experimental study and thermodynamic calculation of the polythermal equilibria. J. Solid State Chem. 2001, 156, 506-511.

64. Kevorkov, D.; Gröbner, J.; Schmid-Fetzer, R. The Al-Li-Si system, Part 1: A new structure type $\mathrm{Li}_{8} \mathrm{Al}_{3} \mathrm{Si}_{5}$ and the ternary solid state phase equilibria. J. Solid State Chem. 2001, 156, 500-505.

65. Zhong, Y.; Ozturk, K.; Liu, Z.K. Thermodynamic modeling of the Ca-Sr-Zn ternary system. J. Phase Equilbria 2003, 24, 340-346.

66. Wang, C.P.; Ohnuma, I.; Kainuma, R.; Ishida, K. Phase equilibria in Fe-Cu-X (X: Co, Cr, Si, V) ternary systems. J. Phase Equilbria 2002, 23, 236-245.

67. An, X.; Li, Q.; Zhang, J.; Chen, S.; Yang, Y. Phase equilibria of the La-Ni-Cu ternary system at $673 \mathrm{~K}$ : Thermodynamic modeling and experimental validation. Calphad 2012, 36, 8-15.

68. Jantzen, T.; Spencer, P.J. Thermodynamic assessment of the $\mathrm{Cu}-\mathrm{Pb}-\mathrm{Zn}$ and $\mathrm{Cu}-\mathrm{Sn}-\mathrm{Zn}$ systems. Calphad 1998, 22, 417-434.

69. Forsberg, A.; Agren, J. Thermodynamic evaluation of the Fe-Mn-Si system and the $\gamma / \varepsilon$ martensitic transformation. J. Phase Equilbria 1993, 14, 354-363.

70. Flandorfer, H.; Gröbner, J.; Stamou, A.; Hasiotis, N.; Saccone, A.; Rogl, P.; Wouters, R.; Seifert, H.J.; Macciò, D.; Ferro, R.; et al. Experimental investigations and thermodynamic calculations in the ternary system Mn-Y-Zr. Z. Metallkd. 1997, 88, 529-538.

71. Janz, A.; Gröbner, J.; Schmid-Fetzer, R. Thermodynamics and constitution of Mg-Al-Ca-Sr-Mn alloys: Part II. Procedure for multicomponent key sample selection and application to the Mg-Al-Ca-Sr and Mg-Al-Ca-Sr-Mn systems. J. Phase Equilbria Diffus. 2009, 30, 157-175.

72. Löffler, A.; Gröbner, J.; Hampl, M.; Engelhardt, H.; Schmid-Fetzer, R.; Rettenmayr, M. Solidifying incongruently melting intermetallic phases as bulk single phases using the example of $\mathrm{Al}_{2} \mathrm{Cu}$ and Q-phase in the Al-Mg-Cu-Si system. J. Alloys Comp. 2012, 515, 123-127.

73. Ohno, M.; Mirkovic, D.; Schmid-Fetzer, R. Liquidus and solidus temperatures of Mg-rich Mg-Al-Mn-Zn alloys. Acta Mater. 2006, 54, 3883-3891.

74. Gröbner, J.; Schmid-Fetzer, R. Selection of promising quaternary candidates from Mg-Mn-(Sc, $\mathrm{Gd}, \mathrm{Y}, \mathrm{Zr}$ ) for development of creep-resistant magnesium alloys. J. Alloys Comp. 2001, 320, 296-301.

75. Bulanova, M.V.; Zheltov, P.N.; Meleshevich, K.A. Lanthanum-cerium-silicon system. J. Alloys Comp. 2002, 347, 149-155.

76. Sinha, V.K.; Wallace, W.E. Hydrogen sorption by the hyperstoichiometric cerium-nickel-copper $\left(\mathrm{Ce} 1+x \mathrm{Ni}_{2.5} \mathrm{Cu}_{2.5}\right)$ alloys. J. Phys. Chem. 1984, 88, 105-107. 
77. Kharchenko, O.I.; Kondratiuk, L.M.; Rak, M.M. Ternary systems (Y,Ce)-Ni-Cu. Vestn. L'vov. Univ. Khim. 1986, 27, 50-52; Available online: http:/www.nbuv.gov.ua/portal/Chem_Biol /Vlnu_kh/index.html (accessed on 9 September 2012).

(C) 2012 by the authors; licensee MDPI, Basel, Switzerland. This article is an open access article distributed under the terms and conditions of the Creative Commons Attribution license (http://creativecommons.org/licenses/by/3.0/). 